\title{
Adoption and implementation of electronic healthcare management system - a bibliometric approach
}

\section{Olayemi Olawumi*}

School of Computing,

University of Eastern Finland,

FI-70211 Kuopio, Finland

Email: olayemo@uef.fi

*Corresponding author

\section{Sunday Olaleye}

Department of Marketing, Management and International Business,

Oulu Business School,

University of Oulu,

90570 Oulu, Finland

Email: sunday.olaleye@oulu.fi

\section{Frank Adusei-Mensah}

Institute of Public Health and Clinical Nutrition,

Faculty of Health Sciences,

University of Eastern Finland,

FI-70211 Kuopio, Finland

Email: franka@uef.fi

\section{Adedayo Olawuni}

Irish College of General Practitioner/Health Service Executive,

Railway Street, Navan, Co. Meath, Ireland

Email: dayolawuni@gmail.com

\section{Richard O. Agjei}

Department of Public Health,

University of Central Nicaragua Medical Center,

Semaforos del Zumen 3C, Nicaragua

Email: richardagjei65@gmail.com

\begin{abstract}
Electronic healthcare management system (EHMS) is seen to have a positive impact on healthcare, but its implementation and adoption are still very low; also, research results on its influence are limited. To ascertain the cause for this gap, a study was conducted to identify gaps in research and knowledge regarding EHMS adoption. This study sought to quantify this lack of research by identifying the current state of EHMS globally and determines how research
\end{abstract}


on implementation, influence, and adoption of EHMS has evolved; two databases were searched for literatures in EHMS, and a bibliometric analysis was performed to understand the nature of research and publication trends in EHMS. We found a relatively small number of literatures that focused on EHMS and a declining state of publication. This study highlights the need to develop a strong evidence base research to support the influence, adoption and effective implementation of EHMS in healthcare institutions.

Keywords: e-health; electronic healthcare management system; EHMS; electronic health record; EHRs; bibliometrics; literature review; citation and co-citation analysis.

Reference to this paper should be made as follows: Olawumi, O., Olaleye, S., Adusei-Mensah, F., Olawuni, A. and Agjei, R.O. (2022) 'Adoption and implementation of electronic healthcare management system - a bibliometric approach', Int. J. Electronic Healthcare, Vol. 12, No. 1, pp.54-96.

Biographical notes: Olayemi Olawumi is a technology risk and security manager. He has extensive experience designing and evaluating IT governance, operational risk, information security for large enterprise environments. $\mathrm{He}$ holds a Doctor of Philosophy and a Master of Science degree in Computer Science from the School of Computing, University of Eastern Finland. His primary research interests include information security, technology risk management, ICT in healthcare research, smart homes, computational intelligence and operation research.

Sunday Olaleye earned his Doctor of Science (D.Sc) in Marketing from the University of Oulu, Oulu Business School (AACSB), Finland. He received his Master of Science in Information Systems from Abo Akademi University, Turku, Finland, MBA from the Lapland University of Applied Sciences, Tornio, Finland, and Certificate of Leadership and Management in Health from the University of Washington, USA. He is currently doing his post-doctoral research at the University of Oulu, Finland, and he is a Visiting Professor at Universidad de las Américas Puebla (UDLAP), Mexico. He has presented papers at conferences and published in academic journals.

Frank Adusei-Mensah is an epidemiologist with broad research experience. He holds PhDs in Public Health and in Epidemiology from University of Eastern Finland and Central University of Nicaragua respectively. He has interest in infectious diseases epidemiology, herbal and functional food research, vaccinology, and electronic health care. He has also carried out research in probiotics and health. He is a Postdoctoral Project Researcher at the Institute of Public Health and Clinical Nutrition, University of Eastern Finland; a researcher at Finnoflag Oy; and Associate Researcher in Public Health at Center for Multidisciplinary Research and Innovations (CMRI), Finland, ORCID: 0000-0001-8237-5305.

Adedayo Olawuni obtained his Doctor of Medicine (MD) degree from the Medical University of Lodz, Poland. He currently works as a consultant family physician, having undergone and completed a four year residency programme in general practice with the Irish College of General Practitioners. He is a member of Irish College of General Practitioners (MICGP), Royal College of Physicians of Ireland (MRCPI), Royal College of Physicians and Surgeon, Glasgow (MRCPS(Glasg)); and a Fellow of both the RSPH and ISQUA. His research interests lie in family and population health, public health and epidemiology, quality improvement in healthcare, information technology and health. 
Richard O. Agjei is currently a student at the Department of Medicine, School of Public Health of Imperial College London. He holds a PhD in Public Health from the Central University of Nicaragua and an MSc in Research Chemistry from the University of Eastern Finland. Additionally, he holds PGCE from Haaga-Helia University of Applied Sciences in Finland. His research interest is multidisciplinary and span across the fields of exposure assessment, affect detection, suicidal behaviours, health policy and analysis, machine learning, and data science applications in public health. He is a reviewer in cogent medicine and has published several articles in scientific journals.

\section{Introduction}

eHealth - the use of information technology for healthcare, is a young and dynamic field that could improve the well-being of people around the world, most notably in Africa and Oceania. Electronic healthcare management system (EHMS) is the application of information technology for health care management. It includes all activities that apply information and communication technology (ICT) to promote healthcare services support, delivery, and education for improving efficiency in health care delivery to the citizens (Adebayo and Ofoegbu, 2014).

Electronics healthcare management system can either be patient care focused, for example, keeping healthcare record for patient care purposes or focused on the administration of healthcare practices. Electronics healthcare management system holds relevant healthcare data that can be used in different healthcare scenarios based on needs. Electronics healthcare management system is an integrated system that can be used in multiple ways in carrying out quality healthcare services for patients, e.g., monitoring of patient's medication and usage over a period of time, significant reduction of paper-based communication of health record and documentation filling through electronic health record (EHRs). The use of EHMS has provided a lot of clinical advantages ranging from the elimination of paper records, easy access to healthcare data, smooth interaction, and collaboration with affiliated healthcare organisations. The emergence and adoption of EHMS into the healthcare sector is on the rise, and it will likely transform the way care is delivered, reduce the cost incurred for healthcare services, and improve the quality of healthcare services provided (Salehahmadi and Hajialiasghari, 2013).

Healthcare institutions around the world, including hospitals, clinics, laboratories and pharmacies, are gradually adopting the use of EHMS into their daily healthcare practices (Evans, 2016). The implementation of electronic health record (EHRs), which is a critical component of EHMS into healthcare institutions, will play a vital role in ensuring healthcare data is collected, stored, and readily available when needed, which will, in turn improve care delivery globally.

Even though EHMS is seen to have positive impact on healthcare, its implementation and adoption is still very low; the technologies have been adopted very slowly by healthcare providers and physicians due to many reasons including the cost of implementation, issues around the usability and support of the new system by healthcare professionals (Ajami and Bagheri-Tadi, 2013). Furthermore, research results and relevant information on the implementation, influence, and adoption of EHMS is limited. In order to ascertain the root cause for this gap, we conducted a study to identify gaps in research and knowledge regarding EHMS adoption and implementation. As part of our study, we 
conduct a comprehensive search of two databases, Web of Science and Scopus, for literature in EHMS. Subsequently, we then embarked on a bibliometric analysis of these kinds of literature to understand the nature of research and publication trends in EHMS globally, taking into consideration some bibliometrics indicators to provide clarity and a different perspective into our study.

Our research will focus on providing answers to the following questions:

1 What is the current state of EHMS globally?

2 How has research on implementation, influence, and adoption of EHMS evolved?

3 What are the landmark research results and publications in EHMS are?

4 What contributions have these EHMS publications had over time?

5 Who has been the most productive and high impact researcher in EHMS?

The rest of the paper is organised as follows; Section 2 discuss in detail our research methodology; Section 3 reviews the bibliometric methods we utilised in this paper. Section 4 presents the results, including the publication and citation trend on EHMS, leading authors, institutions, countries, leading continents, and sources in EHMS research. Section 5 develops a graphical analysis of the bibliographic data of EHMS with VOS viewer software. Section 6 summarises the main findings and concludes the paper.

\section{Research methodology}

In our research, we have used a bibliometric approach to review existing papers in EHMS, following several steps as identified by researchers in Clarke and Horton (2001) and Tranfield et al. (2003). These steps include planning the review, selecting and reviewing the papers, synthesising the results, and reporting the findings (Pérez-Jover et al., 2019).

In planning our literature review, we conducted a comprehensive literature search on two electronic databases: Web of Science and Scopus. We then ranked and grouped the papers for review.

To facilitate the search, we used the following criteria:

1 topic: electronic healthcare management system

2 publication year: 2008-2018

3 document types: articles.

Publications of interest were those published in English and with information on EHMS. Exclusion criteria were studies that were not published in English as well as those that were not related to e-health. We have followed the flowchart below in selecting and reviewing the papers to be included in our study, it explains in detail our methodology from identifying the literature in the two databases to how we arrived at our final selection. 
Figure 1 Flow diagram of inclusion and exclusion (see online version for colours)

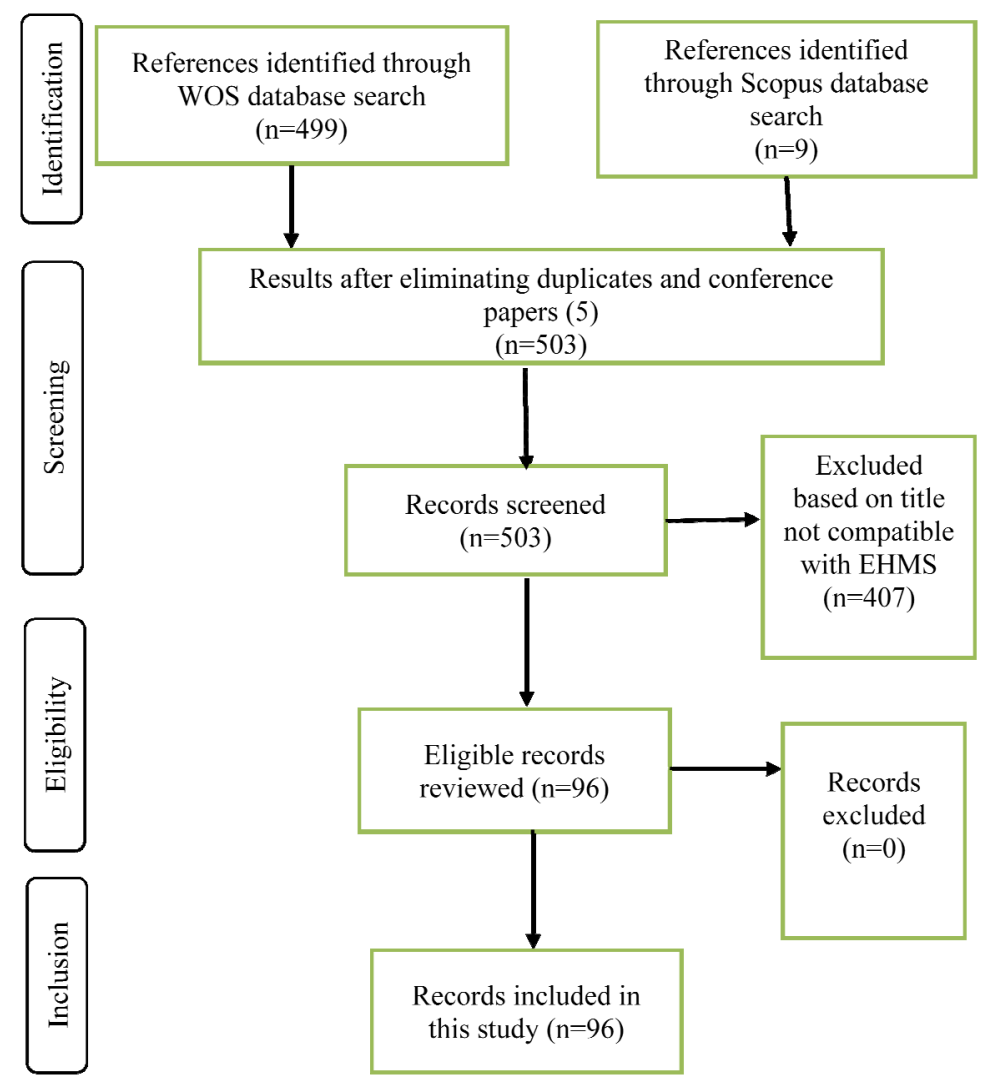

Source: Pérez-Jover et al. (2019)

\section{Applied bibliometric methods in electronic healthcare management system}

Bibliometrics is a research area which applies statistical analysis to bibliometric data to provide understanding, identify patterns or analyse a particular section of literature, and this could include publication in a particular area, authorship, citation (Welsh, 2017; Broadus, 1987; Pritchard, 1969).

Different researchers have categorised bibliometric research in different ways, researchers in Borgman (1989) categorised bibliometrics into three types, which include analysis of the producers, e.g., author, institution; analysis of artefacts, e.g., journals, book chapters, websites; and the analysis of concept e.g., topics or subject areas.

Stevens (1953) took a different approach and categorised bibliometrics into two types, which are descriptive and evaluative. The descriptive approach could take into consideration the productivity of the author, institution, and geographical area while evaluative considers the usage data, citation trend, and h-index.

Nicholas and Ritchie (1978) used two related categories; these include literature characteristics (authorship, year of publication) and literature relationships (citations or 
patterns for co-citing). Another researcher who has categorised differently or develop models to categorise bibliometric research.

In this study, we have taken into consideration most of these indicators to provide clarity and different perspectives to understand our results. Research mainly can be evaluated by productivity and influence (Podsakoff et al., 2008), number of publications in a research area could be used to measure productivity while influence could be measured using the number of citations. In addition to these two indicators, we also considered other bibliometric indicators in our paper including the cites per paper, the cites per year, types of outlets, yearly citation trend, most productive institutions, countries and geographical regions (continents), which was analysed using the number of papers and citations per million inhabitants.

Additionally, our study uses VOSviewer software (van Eck and Waltman, 2009) to graphically map the bibliographic data. VOSviewer is a tool used to analyse bibliometric networks. It works by building maps of authors, publications based on citations, cocitations, co-authorship, co-occurrence of author keywords. All these are key indicators used in analysing our results. Citation analysis is used to identifies how the documents cite each other counting the number of times that article A cites article B and vice versa. When the same third source cites two documents, this is referred to co-citation (Small, 1973).

Co-authorship shows the connections in documents that are co-authored by more than one author, institution or country and lastly, co-occurrence of author keywords identifies the most frequent keywords used by the authors and those keywords that appear more frequently in the same documents.

\section{Results}

We downloaded information about nine articles from Scopus and 499 papers from Web of Science (total 508) that were available by June 2019. The information about the search results from the electronic databases were organised for independent evaluation by our researchers. After performing a critical review of our search results; for example, we eliminated duplicate, performed exclusion based on paper titles not compatible with EHMS and reviewed the publication abstracts, we then arrived at a total of 96 publications to be included in our analysis (91 Web of Science and 5 Scopus papers). We have used the combination of descriptive and evaluative bibliometric approach in carrying out our analysis, which took into consideration productivity of the authors, institution, and geographical area, citation trend etc.; we also used literature relationships including citations and patterns for co-citing as a key indicator to provide clarity to our results.

\subsection{Publication and citation trends}

Table 1 presents the most cited papers in EHMS in Web of Science and Scopus between 2008 and 2018. During this period, the three most cited papers in Web of Science represent the contribution of Sittig, Dean; Greenhalgh, Trisha and Lluch, Maria; who had 171, 143 and 117 respectively. The first two papers by Sittig, Dean; Greenhalgh, Trisha; which are the most cited in Web of Science on EHMS were both published in year 2010. 
Table 1a The most cited papers in EHMS between 2008-2018 (Web of Science)

\begin{tabular}{|c|c|c|c|c|c|}
\hline Order & Author (s) & Title & $\begin{array}{c}\text { Publication } \\
\text { year }\end{array}$ & $\begin{array}{c}\text { Total } \\
\text { citations }\end{array}$ & $\begin{array}{l}\text { Citation } \\
\text { per year }\end{array}$ \\
\hline 1 & $\begin{array}{l}\text { Sittig, Dean F.; } \\
\text { Singh, Hardeep }\end{array}$ & $\begin{array}{l}\text { A new sociotechnical model for } \\
\text { studying health information } \\
\text { technology in complex adaptive } \\
\text { healthcare systems }\end{array}$ & 2010 & 171 & 17.1 \\
\hline 2 & $\begin{array}{l}\text { Greenhalgh, Trisha; } \\
\text { Hinder, Susan; } \\
\text { et al. }\end{array}$ & $\begin{array}{l}\text { Adoption, non-adoption, and } \\
\text { abandonment of a personal } \\
\text { electronic health record: case } \\
\text { study of HealthSpace }\end{array}$ & 2010 & 134 & 13.4 \\
\hline 3 & Lluch, Maria & $\begin{array}{l}\text { Healthcare professionals' } \\
\text { organisational barriers to health } \\
\text { information technologies - } \\
\text { a literature review }\end{array}$ & 2011 & 117 & 13 \\
\hline 4 & $\begin{array}{l}\text { de Lusignan, } \\
\text { Simon; Mold, } \\
\text { Freda; et al. }\end{array}$ & $\begin{array}{l}\text { Patients' online access to their } \\
\text { electronic health records and } \\
\text { linked online services: } \\
\text { a systematic interpretative review }\end{array}$ & 2014 & 73 & 12.17 \\
\hline 5 & $\begin{array}{l}\text { Estabrooks, Paul A.; } \\
\text { Boyle, Maureen; } \\
\text { et al. }\end{array}$ & $\begin{array}{l}\text { Harmonized patient-reported data } \\
\text { elements in the electronic health } \\
\text { record: supporting meaningful use } \\
\text { by primary care action on health } \\
\text { behaviors and key psychosocial } \\
\text { factors }\end{array}$ & 2012 & 72 & 9 \\
\hline 6 & $\begin{array}{l}\text { Mandel, Joshua C.; } \\
\text { Kreda, David A.; } \\
\text { et al. }\end{array}$ & $\begin{array}{l}\text { SMART on FHIR: } \\
\text { a standards-based, interoperable } \\
\text { apps platform for electronic health } \\
\text { records }\end{array}$ & 2016 & 57 & 14.25 \\
\hline 7 & $\begin{array}{l}\text { Kazley, Abby S.; } \\
\text { Ozcan, Yasar A. }\end{array}$ & $\begin{array}{c}\text { Do hospitals with electronic } \\
\text { medical records (EMRs) provide } \\
\text { higher quality care? An } \\
\text { examination of three clinical } \\
\text { conditions }\end{array}$ & 2008 & 52 & 4.33 \\
\hline 8 & $\begin{array}{l}\text { Masys, Daniel R.; } \\
\text { Jarvik, Gail P.; } \\
\text { et al. }\end{array}$ & $\begin{array}{l}\text { Technical desiderata for the } \\
\text { integration of genomic data into } \\
\text { electronic health records }\end{array}$ & 2012 & 51 & 6.38 \\
\hline 9 & $\begin{array}{l}\text { Peleg, Mor; Keren, } \\
\text { Sagi; Denekamp, } \\
\text { Yaron }\end{array}$ & $\begin{array}{l}\text { Mapping computerized clinical } \\
\text { guidelines to electronic medical } \\
\text { records: knowledge-data } \\
\text { ontological mapper (KDOM) }\end{array}$ & 2008 & 51 & 4.25 \\
\hline 10 & $\begin{array}{l}\text { Terry, Amanda L.; } \\
\text { Thorpe, Cathy F.; } \\
\text { et al. }\end{array}$ & $\begin{array}{l}\text { Implementing electronic health } \\
\text { records - key factors in primary } \\
\text { care }\end{array}$ & 2008 & 49 & 4.08 \\
\hline 11 & $\begin{array}{l}\text { Wright, Adam; } \\
\text { Poon, Eric G.; } \\
\quad \text { et al. }\end{array}$ & $\begin{array}{l}\text { Randomized controlled trial of } \\
\text { health maintenance reminders } \\
\text { provided directly to patients } \\
\text { through an electronic PHR }\end{array}$ & 2012 & 48 & 6 \\
\hline
\end{tabular}


Table 1a The most cited papers in EHMS between 2008-2018 (Web of Science) (continued)

\begin{tabular}{|c|c|c|c|c|c|}
\hline Order & Author (s) & Title & $\begin{array}{c}\text { Publication } \\
\text { year }\end{array}$ & $\begin{array}{c}\text { Total } \\
\text { citations }\end{array}$ & $\begin{array}{l}\text { Citation } \\
\text { per year }\end{array}$ \\
\hline 12 & $\begin{array}{l}\text { Stoves, John; } \\
\text { Connolly, John; } \\
\text { et al. }\end{array}$ & $\begin{array}{l}\text { Electronic consultation as an } \\
\text { alternative to hospital referral for } \\
\text { patients with chronic kidney } \\
\text { disease: a novel application for } \\
\text { networked electronic health } \\
\text { records to improve the } \\
\text { accessibility and efficiency of } \\
\text { healthcare }\end{array}$ & 2010 & 45 & 4.5 \\
\hline 13 & $\begin{array}{l}\text { Wong, Martin C.S.; } \\
\text { Jiang, Johnny Y.; } \\
\text { et al. }\end{array}$ & $\begin{array}{l}\text { Health services research in the } \\
\text { public healthcare system in } \\
\text { Hong Kong: an analysis of over } \\
1 \text { million antihypertensive } \\
\text { prescriptions between 2004-2007 } \\
\text { as an example of the potential and } \\
\text { pitfalls of using routinely } \\
\text { collected electronic patient data }\end{array}$ & 2008 & 45 & 3.75 \\
\hline 14 & $\begin{array}{l}\text { Freeman, R.; } \\
\text { Moore, L.S.P.; et al. }\end{array}$ & $\begin{array}{c}\text { Advances in electronic } \\
\text { surveillance for } \\
\text { healthcare-associated infections in } \\
\text { the } 21 \text { st Century: a systematic } \\
\text { review }\end{array}$ & 2013 & 43 & 6.14 \\
\hline 15 & $\begin{array}{l}\text { Rothman, Brian; } \\
\text { Leonard, Joan C.; } \\
\text { Vigoda, Michael M. }\end{array}$ & $\begin{array}{l}\text { Future of electronic health } \\
\text { records: implications for decision } \\
\text { support }\end{array}$ & 2012 & 38 & 4.75 \\
\hline 16 & $\begin{array}{l}\text { Ng, Kenney; } \\
\text { Ghoting, Amol; } \\
\text { et al. }\end{array}$ & $\begin{array}{l}\text { PARAMO: a parallel predictive } \\
\text { modeling platform for healthcare } \\
\text { analytic research using electronic } \\
\text { health records }\end{array}$ & 2014 & 36 & 6 \\
\hline 17 & $\begin{array}{l}\text { Chen, Yu-Yi; Lu, } \\
\text { Jun-Chao; Jan, } \\
\text { Jinn-Ke }\end{array}$ & $\begin{array}{c}\text { A secure EHR system based on } \\
\text { hybrid clouds }\end{array}$ & 2012 & 35 & 4.38 \\
\hline 18 & $\begin{array}{c}\text { Linder, Jeffrey A.; } \\
\text { Kaleba, Erin O.; } \\
\text { Kmetik, Karen S. }\end{array}$ & $\begin{array}{l}\text { Using electronic health records to } \\
\text { measure physician performance } \\
\text { for acute conditions in primary } \\
\text { care empirical evaluation of the } \\
\text { community-acquired pneumonia } \\
\text { clinical quality measure set }\end{array}$ & 2009 & 32 & 2.91 \\
\hline 19 & $\begin{array}{l}\text { Meeks, Derek W.; } \\
\text { Takian, } \\
\text { Amirhossein; et al. }\end{array}$ & $\begin{array}{l}\text { Exploring the sociotechnical } \\
\text { intersection of patient safety and } \\
\text { electronic health record } \\
\text { implementation }\end{array}$ & 2014 & 31 & 5.17 \\
\hline 20 & $\begin{array}{l}\text { Wright, Adam; } \\
\text { Sittig, Dean F.; } \\
\text { et al. }\end{array}$ & $\begin{array}{l}\text { Development and evaluation of a } \\
\text { comprehensive clinical decision } \\
\text { support taxonomy: comparison of } \\
\text { front-end tools in commercial and } \\
\text { internally developed electronic } \\
\text { health record systems }\end{array}$ & 2011 & 30 & 3.33 \\
\hline
\end{tabular}


Table 1a The most cited papers in EHMS between 2008-2018 (Web of Science) (continued)

\begin{tabular}{|c|c|c|c|c|c|}
\hline Order & Author (s) & Title & $\begin{array}{l}\text { Publication } \\
\text { year }\end{array}$ & $\begin{array}{c}\text { Total } \\
\text { citations }\end{array}$ & $\begin{array}{l}\text { Citation } \\
\text { per year }\end{array}$ \\
\hline 21 & $\begin{array}{l}\text { Hsu, William; Taira, } \\
\text { Ricky K.; et al. }\end{array}$ & $\begin{array}{l}\text { Context-based electronic health } \\
\text { record: toward patient specific } \\
\text { healthcare }\end{array}$ & 2012 & 29 & 3.63 \\
\hline 22 & $\begin{array}{l}\text { Morley, Katherine } \\
\text { I.; Wallace, Joshua; } \\
\text { et al. }\end{array}$ & $\begin{array}{l}\text { Defining disease phenotypes } \\
\text { using national linked electronic } \\
\text { health records: a case study of } \\
\text { atrial fibrillation }\end{array}$ & 2014 & 28 & 4.67 \\
\hline 23 & $\begin{array}{l}\text { Mishra, Abhay } \\
\text { Nath; Anderson, } \\
\text { Catherine; } \\
\text { et al. }\end{array}$ & $\begin{array}{c}\text { Electronic health records } \\
\text { assimilation and physician } \\
\text { identity evolution: an identity } \\
\text { theory perspective }\end{array}$ & 2012 & 27 & 3.38 \\
\hline 24 & $\begin{array}{l}\text { Harris, Stewart B.; } \\
\text { Glazier, Richard H.; } \\
\text { et al. }\end{array}$ & $\begin{array}{l}\text { Investigating concordance in } \\
\text { diabetes diagnosis between } \\
\text { primary care charts (electronic } \\
\text { medical records) and health } \\
\text { administrative data: a } \\
\text { retrospective cohort study }\end{array}$ & 2010 & 27 & 2.7 \\
\hline 25 & $\begin{array}{c}\text { Sittig, Dean F.; } \\
\text { Wright, Adam; et al. }\end{array}$ & $\begin{array}{c}\text { Comparison of clinical knowledge } \\
\text { management capabilities of } \\
\text { commercially-available and } \\
\text { leading internally-developed } \\
\text { electronic health records }\end{array}$ & 2011 & 26 & 2.89 \\
\hline 26 & $\begin{array}{l}\text { Cho, InSook; Kim, } \\
\text { JeongAh; } \\
\text { et al. }\end{array}$ & $\begin{array}{l}\text { Design and implementation of a } \\
\text { standards-based interoperable } \\
\text { clinical decision support } \\
\text { architecture in the context of the } \\
\text { Korean HER }\end{array}$ & 2010 & 25 & 2.5 \\
\hline 27 & $\begin{array}{l}\text { Pahl, Christina; } \\
\text { Zare, Mojtaba; } \\
\quad \text { et al. }\end{array}$ & $\begin{array}{l}\text { Role of OpenEHR as an open } \\
\text { source solution for the regional } \\
\text { modelling of patient data in } \\
\text { obstetrics }\end{array}$ & 2015 & 23 & 4.6 \\
\hline 28 & $\begin{array}{l}\text { Ancker, Jessica S.; } \\
\text { Kern, Lisa M.; et al. }\end{array}$ & $\begin{array}{l}\text { How is the electronic health } \\
\text { record being used? Use of EHR } \\
\text { data to assess physician-level } \\
\text { variability in technology use }\end{array}$ & 2014 & 23 & 3.83 \\
\hline 29 & $\begin{array}{l}\text { Saleem, Jason J.; } \\
\text { Flanagan, Mindy E.; } \\
\text { et al. }\end{array}$ & $\begin{array}{l}\text { The next-generation electronic } \\
\text { health record: perspectives of key } \\
\text { leaders from the US Department } \\
\text { of Veterans Affairs }\end{array}$ & 2013 & 23 & 3.29 \\
\hline 30 & $\begin{array}{l}\text { McGinn, Carrie } \\
\text { Anna; Gagnon, } \\
\text { Marie-Pierre; et al. }\end{array}$ & $\begin{array}{l}\text { Users' perspectives of key factors } \\
\text { to implementing electronic health } \\
\text { records in Canada: a Delphi study }\end{array}$ & 2012 & 23 & 2.88 \\
\hline 31 & $\begin{array}{l}\text { Jarvis, Benjamin; } \\
\text { Johnson, Tricia; et } \\
\text { al. }\end{array}$ & $\begin{array}{c}\text { Assessing the impact of electronic } \\
\text { health records as an enabler of } \\
\text { hospital quality and patient } \\
\text { satisfaction }\end{array}$ & 2013 & 22 & 3.14 \\
\hline
\end{tabular}


Table 1a The most cited papers in EHMS between 2008-2018 (Web of Science) (continued)

\begin{tabular}{|c|c|c|c|c|c|}
\hline Order & Author (s) & Title & $\begin{array}{l}\text { Publication } \\
\text { year }\end{array}$ & $\begin{array}{c}\text { Total } \\
\text { citations }\end{array}$ & $\begin{array}{l}\text { Citation } \\
\text { per year }\end{array}$ \\
\hline 32 & Sassen, Elizabeth J. & $\begin{array}{l}\text { Love, hate, or indifference how } \\
\text { nurses really feel about the } \\
\text { electronic health record system }\end{array}$ & 2009 & 22 & 2 \\
\hline 33 & $\begin{array}{l}\text { O'Reilly, Daria; } \\
\text { Holbrook, Anne; } \\
\quad \text { et al. }\end{array}$ & $\begin{array}{l}\text { Cost-effectiveness of a shared } \\
\text { computerized decision support } \\
\text { system for diabetes linked to } \\
\text { electronic medical records }\end{array}$ & 2012 & 21 & 2.63 \\
\hline 34 & $\begin{array}{l}\text { Kumar, Rajiv B.; } \\
\text { Goren, Nira D.; et } \\
\text { al. }\end{array}$ & $\begin{array}{l}\text { Automated integration of } \\
\text { continuous glucose monitor data } \\
\text { in the electronic health record } \\
\text { using consumer technology }\end{array}$ & 2016 & 20 & 5 \\
\hline 35 & $\begin{array}{l}\text { Wang, } \\
\text { Hua-Qiong; Li, } \\
\text { Jing-Song; } \\
\text { et al. }\end{array}$ & $\begin{array}{l}\text { Creating personalised clinical } \\
\text { pathways by semantic } \\
\text { interoperability with electronic } \\
\text { health records }\end{array}$ & 2013 & 20 & 2.86 \\
\hline 36 & Benhamou, P-Y. & $\begin{array}{l}\text { Improving diabetes management } \\
\text { with electronic health records and } \\
\text { patients' health records }\end{array}$ & 2011 & 20 & 2.22 \\
\hline 37 & $\begin{array}{l}\text { Prociow, Pawel A.; } \\
\text { Crowe, John A. }\end{array}$ & $\begin{array}{c}\text { Towards personalised ambient } \\
\text { monitoring of mental health via } \\
\text { mobile technologies }\end{array}$ & 2010 & 20 & 2 \\
\hline 38 & $\begin{array}{l}\text { Rahimi, Alireza; } \\
\text { Liaw, } \\
\text { Siaw-Teng; et al. }\end{array}$ & $\begin{array}{l}\text { Validating an ontology-based } \\
\text { algorithm to identify patients with } \\
\text { type } 2 \text { diabetes mellitus in } \\
\text { electronic health records }\end{array}$ & 2014 & 19 & 3.17 \\
\hline 39 & $\begin{array}{l}\text { Herbek, S.; Eisl, } \\
\text { H.A.; } \\
\text { et al. }\end{array}$ & $\begin{array}{l}\text { The electronic health record in } \\
\text { Austria: a strong network between } \\
\text { health care and patients }\end{array}$ & 2012 & 19 & 2.38 \\
\hline 40 & $\begin{array}{l}\text { Wright, Adam; } \\
\text { McCoy, Allison B.; } \\
\text { et al. }\end{array}$ & $\begin{array}{l}\text { Problem list completeness in } \\
\text { electronic health records: a multi- } \\
\text { site study and assessment of } \\
\text { success factors }\end{array}$ & 2015 & 18 & 3.6 \\
\hline 41 & $\begin{array}{l}\text { Phansalkar, Shobha; } \\
\text { Zachariah, } \\
\text { Marianne; } \\
\text { et al. }\end{array}$ & $\begin{array}{l}\text { Evaluation of medication alerts in } \\
\text { electronic health records for } \\
\text { compliance with human factors } \\
\text { principles }\end{array}$ & 2014 & 18 & 3 \\
\hline 42 & $\begin{array}{l}\text { Kopanitsa, G.; } \\
\text { Hildebrand, C.; et } \\
\text { al. }\end{array}$ & $\begin{array}{l}\text { Visualization of medical data } \\
\text { based on EHR standards }\end{array}$ & 2013 & 18 & 2.57 \\
\hline 43 & $\begin{array}{l}\text { Banerjee, Dipanjan; } \\
\text { Chung, Sukyung; } \\
\text { et al. }\end{array}$ & $\begin{array}{l}\text { Underdiagnosis of Hypertension } \\
\text { Using Electronic Health Records }\end{array}$ & 2012 & 18 & 2.25 \\
\hline 44 & $\begin{array}{l}\text { Li, } \\
\text { Jing-Song; Zhang, } \\
\text { Xiao-Guang; et al. }\end{array}$ & $\begin{array}{l}\text { The meaningful use of EMR in } \\
\text { Chinese hospitals: a case study on } \\
\text { curbing antibiotic abuse }\end{array}$ & 2013 & 17 & 2.43 \\
\hline
\end{tabular}


Table 1a The most cited papers in EHMS between 2008-2018 (Web of Science) (continued)

\begin{tabular}{|c|c|c|c|c|c|}
\hline Order & Author (s) & Title & $\begin{array}{c}\text { Publication } \\
\text { year }\end{array}$ & $\begin{array}{c}\text { Total } \\
\text { citations }\end{array}$ & $\begin{array}{l}\text { Citation } \\
\text { per year }\end{array}$ \\
\hline 45 & $\begin{array}{l}\text { Menon, Shailaja; } \\
\text { Smith, Michael W.; } \\
\text { et al. }\end{array}$ & $\begin{array}{l}\text { How context affects electronic } \\
\text { health record-based test result } \\
\text { follow-up: } \\
\text { a mixed-methods evaluation }\end{array}$ & 2014 & 16 & 2.67 \\
\hline 46 & $\begin{array}{l}\text { Noblin, Alice; } \\
\text { Cortelyou-Ward, } \\
\text { Kendall; } \\
\text { et al. }\end{array}$ & $\begin{array}{l}\text { EHR implementation in a new } \\
\text { clinic: } \\
\text { a case study of clinician } \\
\text { perceptions }\end{array}$ & 2013 & 16 & 2.29 \\
\hline 47 & $\begin{array}{l}\text { Makam, Anil N.; } \\
\text { Nguyen, Oanh K.; } \\
\text { et al. }\end{array}$ & $\begin{array}{l}\text { Identifying patients with diabetes } \\
\text { and the earliest date of diagnosis } \\
\text { in real time: an electronic health } \\
\text { record case-finding algorithm }\end{array}$ & 2013 & 15 & 2.14 \\
\hline 48 & $\begin{array}{l}\text { Zhang, Jianguo; } \\
\text { Zhang, Kai; et al. }\end{array}$ & $\begin{array}{l}\text { Grid-based implementation of } \\
\text { XDS-I as part of image-enabled } \\
\text { EHR for regional healthcare in } \\
\text { Shanghai }\end{array}$ & 2011 & 15 & 1.67 \\
\hline 49 & $\begin{array}{l}\text { Ancker, Jessica S.; } \\
\text { Kern, Lisa M.; et al. }\end{array}$ & $\begin{array}{l}\text { Associations between healthcare } \\
\text { quality and use of electronic } \\
\text { health record functions in } \\
\text { ambulatory care }\end{array}$ & 2015 & 14 & 2.8 \\
\hline 50 & $\begin{array}{l}\text { Guo, Rui; Shi, } \\
\text { Huixian; et al. }\end{array}$ & $\begin{array}{l}\text { Secure attribute-based signature } \\
\text { scheme with multiple authorities } \\
\text { for blockchain in electronic health } \\
\text { records systems }\end{array}$ & 2018 & 13 & 6.5 \\
\hline 51 & $\begin{array}{l}\text { Dagher, Gaby G.; } \\
\text { Mohler, Jordan; et } \\
\quad \text { al. }\end{array}$ & $\begin{array}{c}\text { Ancile: privacy-preserving } \\
\text { framework for access control and } \\
\text { interoperability of electronic } \\
\text { health records using blockchain } \\
\text { technology }\end{array}$ & 2018 & 12 & 6 \\
\hline 52 & $\begin{array}{l}\text { Goldstein, David } \\
\text { H.; Phelan, Rachel; } \\
\text { et al. }\end{array}$ & $\begin{array}{l}\text { Brief review: Adoption of } \\
\text { electronic medical records to } \\
\text { enhance acute pain management }\end{array}$ & 2014 & 12 & 2 \\
\hline 53 & $\begin{array}{l}\text { Teufel, Ronald J., } \\
\text { II; Kazley, Abby } \\
\text { Swanson; } \\
\text { et al. }\end{array}$ & $\begin{array}{c}\text { Hospital electronic medical record } \\
\text { use and cost of inpatient pediatric } \\
\text { care }\end{array}$ & 2012 & 12 & 1.5 \\
\hline 54 & $\begin{array}{l}\text { Li, Jing-Song; } \\
\text { Zhang, Xiao-Guang; } \\
\text { et al. }\end{array}$ & $\begin{array}{c}\text { Design and development of EMR } \\
\text { supporting medical process } \\
\text { management }\end{array}$ & 2012 & 12 & 1.5 \\
\hline 55 & $\begin{array}{l}\text { Newsham, } \\
\text { Alexander C.; } \\
\text { Johnston, Colin; } \\
\quad \text { et al. }\end{array}$ & $\begin{array}{l}\text { Development of an advanced } \\
\text { database for clinical trials } \\
\text { integrated with an electronic } \\
\text { patient record system }\end{array}$ & 2011 & 12 & 1.33 \\
\hline 56 & $\begin{array}{l}\text { Xiao, Liang; } \\
\text { Cousins, Grainne; } \\
\text { et al. }\end{array}$ & $\begin{array}{l}\text { Developing an electronic health } \\
\text { record (EHR) for methadone } \\
\text { treatment recording and decision } \\
\text { support }\end{array}$ & 2011 & 12 & 1.33 \\
\hline
\end{tabular}


Table 1a The most cited papers in EHMS between 2008-2018 (Web of Science) (continued)

\begin{tabular}{|c|c|c|c|c|c|}
\hline Order & Author (s) & Title & $\begin{array}{l}\text { Publication } \\
\text { year }\end{array}$ & $\begin{array}{c}\text { Total } \\
\text { citations }\end{array}$ & $\begin{array}{l}\text { Citation } \\
\text { per year }\end{array}$ \\
\hline 57 & $\begin{array}{l}\text { Marimon-Sunol, } \\
\text { Santiago; } \\
\text { Rovira-Barbera, } \\
\text { Maria; et al. }\end{array}$ & $\begin{array}{c}\text { Shared electronic health record in } \\
\text { Catalonia, Spain }\end{array}$ & 2010 & 11 & 1.1 \\
\hline 58 & $\begin{array}{l}\text { Grant, C.; } \\
\text { Ludbrook, G.; } \\
\quad \text { et al. }\end{array}$ & $\begin{array}{c}\text { Adverse physiological events } \\
\text { under anaesthesia and sedation: a } \\
\text { pilot audit of electronic patient } \\
\text { records }\end{array}$ & 2008 & 11 & 0.92 \\
\hline 59 & $\begin{array}{c}\text { Yadav, Pranjul; } \\
\text { Steinbach, Michael; } \\
\text { et al. }\end{array}$ & $\begin{array}{l}\text { Mining electronic health records } \\
\text { (EHRs): a survey }\end{array}$ & 2018 & 10 & 5 \\
\hline 60 & $\begin{array}{l}\text { van Velthoven, } \\
\text { Michelle Helena; } \\
\text { Mastellos, Nikolaos; } \\
\text { et al. }\end{array}$ & $\begin{array}{l}\text { Feasibility of extracting data from } \\
\text { electronic medical records for } \\
\text { research: an international } \\
\text { comparative study }\end{array}$ & 2016 & 10 & 2.5 \\
\hline 61 & $\begin{array}{l}\text { Liu, Chung-Feng; } \\
\text { Cheng, Tain-Junn }\end{array}$ & $\begin{array}{c}\text { Exploring critical factors } \\
\text { influencing physicians' } \\
\text { acceptance of mobile electronic } \\
\text { medical records based on the } \\
\text { dual-factor model: a validation in } \\
\text { Taiwan }\end{array}$ & 2015 & 10 & 2 \\
\hline 62 & $\begin{array}{l}\text { Shea, Christopher } \\
\text { M.; Reiter, Kristin } \\
\text { L.; et al. }\end{array}$ & $\begin{array}{l}\text { Stage } 1 \text { of the meaningful use } \\
\text { incentive program for electronic } \\
\text { health records: a study of } \\
\text { readiness for change in } \\
\text { ambulatory practice settings in } \\
\text { one integrated delivery system }\end{array}$ & 2014 & 10 & 1.67 \\
\hline 63 & $\begin{array}{l}\text { Kontio, Elina; } \\
\text { Airola, Antti; } \\
\text { et al. }\end{array}$ & $\begin{array}{l}\text { Predicting patient acuity from } \\
\text { electronic patient records }\end{array}$ & 2014 & 10 & 1.67 \\
\hline 64 & $\begin{array}{l}\text { Heart, Tsipi; } \\
\text { Ben-Assuli, Ofir; } \\
\text { Shabtai, Itamar }\end{array}$ & $\begin{array}{c}\text { A review of PHR, EMR and EHR } \\
\text { integration: A more personalized } \\
\text { healthcare and public health } \\
\text { policy }\end{array}$ & 2017 & 9 & 3 \\
\hline 65 & $\begin{array}{l}\text { Jawhari, Badeia; } \\
\text { Keenan, Louanne; } \\
\text { et al. }\end{array}$ & $\begin{array}{l}\text { Barriers and facilitators to } \\
\text { Electronic Medical Record } \\
\text { (EMR) use in an urban slum }\end{array}$ & 2016 & 9 & 2.25 \\
\hline 66 & $\begin{array}{l}\text { Gagnon, } \\
\text { Marie-Pierre; } \\
\text { Payne-Gagnon, } \\
\text { Julie; et al. }\end{array}$ & $\begin{array}{l}\text { Adoption of electronic personal } \\
\text { health records in Canada: } \\
\text { perceptions of stakeholders }\end{array}$ & 2016 & 9 & 2.25 \\
\hline 67 & $\begin{array}{l}\text { McAlearney, Ann } \\
\text { Scheck; Hefner, } \\
\text { Jennifer L.; et al. }\end{array}$ & $\begin{array}{l}\text { Evidence-based management of } \\
\text { ambulatory electronic health } \\
\text { record system implementation: } \\
\text { An assessment of conceptual } \\
\text { support and qualitative evidence }\end{array}$ & 2014 & 9 & 1.5 \\
\hline
\end{tabular}


Table 1a The most cited papers in EHMS between 2008-2018 (Web of Science) (continued)

\begin{tabular}{|c|c|c|c|c|c|}
\hline Order & Author (s) & Title & $\begin{array}{l}\text { Publication } \\
\text { year }\end{array}$ & $\begin{array}{c}\text { Total } \\
\text { citations }\end{array}$ & $\begin{array}{l}\text { Citation } \\
\text { per year }\end{array}$ \\
\hline 68 & $\begin{array}{c}\text { Tundia, Namita L.; } \\
\text { Kelton, Christina M. } \\
\text { L.; et al. }\end{array}$ & $\begin{array}{l}\text { The effect of electronic medical } \\
\text { record system sophistication on } \\
\text { preventive healthcare for women }\end{array}$ & 2013 & 9 & 1.29 \\
\hline 69 & $\begin{array}{l}\text { Navarro, Ronald A.; } \\
\text { Greene, Denise F.; } \\
\text { et al. }\end{array}$ & $\begin{array}{c}\text { Minimizing Disparities in } \\
\text { Osteoporosis Care of Minorities } \\
\text { with an Electronic Medical } \\
\text { Record Care Plan }\end{array}$ & 2011 & 9 & 1 \\
\hline 70 & $\begin{array}{l}\text { del Carmen } \\
\text { Legaz-Garcia, } \\
\text { Maria; } \\
\text { Martinez-Costa, } \\
\text { Catalina; et al. }\end{array}$ & $\begin{array}{l}\text { A semantic web-based framework } \\
\text { for the interoperability and } \\
\text { exploitation of clinical models } \\
\text { and EHR data }\end{array}$ & 2016 & 8 & 2 \\
\hline 71 & $\begin{array}{l}\text { Sajatovic, Martha; } \\
\text { Welter, Elisabeth; } \\
\quad \text { et al. }\end{array}$ & $\begin{array}{c}\text { Electronic medical record analysis } \\
\text { of emergency room visits and } \\
\text { hospitalizations in individuals } \\
\text { with epilepsy and mental illness } \\
\text { comorbidity }\end{array}$ & 2015 & 8 & 1.6 \\
\hline 72 & $\begin{array}{l}\text { Park, Sun Young; } \\
\text { Chen, Yunan; } \\
\text { Rudkin, Scott }\end{array}$ & $\begin{array}{l}\text { Technological and organizational } \\
\text { adaptation of EMR } \\
\text { implementation in an emergency } \\
\text { department }\end{array}$ & 2015 & 8 & 1.6 \\
\hline 73 & $\begin{array}{l}\text { Cardoso de Moraes, } \\
\text { Joao Luis; de Souza, } \\
\text { Wanderley Lopes; } \\
\text { et al. }\end{array}$ & $\begin{array}{l}\text { A methodology based on } \\
\text { openEHR archetypes and } \\
\text { software agents for developing } \\
\text { e-health applications reusing } \\
\text { legacy systems }\end{array}$ & 2016 & 7 & 1.75 \\
\hline 74 & $\begin{array}{l}\text { Teufel, Ronald J., } \\
\text { II; Kazley, Abby } \\
\text { Swanson; et al. }\end{array}$ & $\begin{array}{l}\text { Electronic medical record } \\
\text { adoption in hospitals that care for } \\
\text { children }\end{array}$ & 2013 & 7 & 1 \\
\hline 75 & $\begin{array}{l}\text { Al Mallah, Amr; } \\
\text { Guelpa, Paul; et al. }\end{array}$ & $\begin{array}{l}\text { Integrating genomic-based } \\
\text { clinical decision support into } \\
\text { electronic health records }\end{array}$ & 2010 & 7 & 0.7 \\
\hline 76 & $\begin{array}{l}\text { Kharrazi, Hadi; } \\
\text { Gonzalez, Claudia } \\
\text { P.; et al. }\end{array}$ & $\begin{array}{l}\text { Forecasting the maturation of } \\
\text { electronic health record functions } \\
\text { among US hospitals: retrospective } \\
\text { analysis and predictive model }\end{array}$ & 2018 & 6 & 3 \\
\hline 77 & $\begin{array}{l}\text { Plantier, Morgane; } \\
\text { Havet, Nathalie; } \\
\text { et al. }\end{array}$ & $\begin{array}{l}\text { Does adoption of electronic health } \\
\text { records improve the quality of } \\
\text { care management in France? } \\
\text { Results from the French e-SI } \\
\text { (PREPS-SIPS) study }\end{array}$ & 2017 & 6 & 2 \\
\hline 78 & $\begin{array}{l}\text { de Ruiter, } \\
\text { Hans-Peter; } \\
\text { Liaschenko, Joan; } \\
\text { Angus, Jan }\end{array}$ & $\begin{array}{l}\text { Problems with the electronic } \\
\text { health record }\end{array}$ & 2016 & 6 & 1.5 \\
\hline
\end{tabular}


Table 1a The most cited papers in EHMS between 2008-2018 (Web of Science) (continued)

\begin{tabular}{|c|c|c|c|c|c|}
\hline Order & Author (s) & Title & $\begin{array}{c}\text { Publication } \\
\text { year }\end{array}$ & $\begin{array}{c}\text { Total } \\
\text { citations }\end{array}$ & $\begin{array}{l}\text { Citation } \\
\text { per year }\end{array}$ \\
\hline 79 & $\begin{array}{l}\text { Somolinos, Roberto; } \\
\text { Munoz, Adolfo; } \\
\text { et al. }\end{array}$ & $\begin{array}{l}\text { Service for the pseudonymization } \\
\text { of electronic healthcare records } \\
\text { based on ISO/EN } 13606 \text { for the } \\
\text { secondary use of information }\end{array}$ & 2015 & 6 & 1.2 \\
\hline 80 & $\begin{array}{l}\text { McCoy, Allison B.; } \\
\text { Wright, Adam; } \\
\text { Sittig, Dean F. }\end{array}$ & $\begin{array}{l}\text { Cross-vendor evaluation of key } \\
\text { user-defined clinical decision } \\
\text { support capabilities: a } \\
\text { scenario-based assessment of } \\
\text { certified electronic health records } \\
\text { with guidelines for future } \\
\text { development }\end{array}$ & 2015 & 6 & 1.2 \\
\hline 81 & $\begin{array}{c}\text { Marceglia, S.; } \\
\text { Fontelo, P.; et al. }\end{array}$ & $\begin{array}{l}\text { A standards-based architecture } \\
\text { proposal for integrating patient } \\
\text { mHealth apps to electronic health } \\
\text { record systems }\end{array}$ & 2015 & 6 & 1.2 \\
\hline 82 & $\begin{array}{l}\text { Hazlehurst, Brian } \\
\text { L.; Lawrence, Jean } \\
\text { M.; et al. }\end{array}$ & $\begin{array}{l}\text { Automating assessment of } \\
\text { lifestyle counseling in electronic } \\
\text { health records }\end{array}$ & 2014 & 6 & 1 \\
\hline 83 & $\begin{array}{l}\text { Zaninelli, M.; } \\
\text { Campagnoli, A.; } \\
\text { et al. }\end{array}$ & $\begin{array}{l}\text { The O3-Vet project: Integration of } \\
\text { a standard nomenclature of } \\
\text { clinical terms in a veterinary } \\
\text { electronic medical record for } \\
\text { veterinary hospitals }\end{array}$ & 2012 & 6 & 0.75 \\
\hline 84 & $\begin{array}{l}\text { McEwen, Timothy } \\
\text { R.; Elder, Nancy C.; } \\
\text { Flach, John M. }\end{array}$ & $\begin{array}{l}\text { Creating safety in primary care } \\
\text { practice with electronic medical } \\
\text { records requires the consideration } \\
\text { of system dynamics }\end{array}$ & 2011 & 6 & 0.67 \\
\hline 85 & $\begin{array}{l}\text { De Pietro, Carlo; } \\
\text { Francetic, Igor }\end{array}$ & $\begin{array}{l}\text { E-health in Switzerland: } \\
\text { the laborious adoption of the } \\
\text { federal law on electronic health } \\
\text { records (EHR) and health } \\
\text { information exchange (HIE) } \\
\text { networks }\end{array}$ & 2018 & 5 & 2.5 \\
\hline 86 & $\begin{array}{l}\text { Wang, Shirley V.; } \\
\text { Rogers, James R.; } \\
\quad \text { et al. }\end{array}$ & $\begin{array}{l}\text { Use of electronic healthcare } \\
\text { records to identify complex } \\
\text { patients with atrial fibrillation for } \\
\text { targeted intervention }\end{array}$ & 2017 & 5 & 1.67 \\
\hline 87 & $\begin{array}{l}\text { Matton, Marie-Pier; } \\
\text { Toledano, Baruch; } \\
\text { et al. }\end{array}$ & $\begin{array}{l}\text { Electronic medical record in } \\
\text { pediatric intensive care: } \\
\text { implementation process } \\
\text { assessment }\end{array}$ & 2016 & 5 & 1.25 \\
\hline 88 & $\begin{array}{l}\text { Kafi, Mohamed } \\
\text { Amine; Ben } \\
\text { Othman, Jalel; } \\
\text { et al. }\end{array}$ & $\begin{array}{l}\text { CCS_WHMS: a congestion } \\
\text { control scheme for wearable } \\
\text { health management system }\end{array}$ & 2015 & 5 & 1 \\
\hline
\end{tabular}


Table 1a The most cited papers in EHMS between 2008-2018 (Web of Science) (continued)

\begin{tabular}{|c|c|c|c|c|c|}
\hline Order & Author (s) & Title & $\begin{array}{l}\text { Publication } \\
\text { year }\end{array}$ & $\begin{array}{c}\text { Total } \\
\text { citations }\end{array}$ & $\begin{array}{l}\text { Citation } \\
\text { per year }\end{array}$ \\
\hline 89 & $\begin{array}{l}\text { Petrakaki, Dimitra; } \\
\text { Klecun, Ela }\end{array}$ & $\begin{array}{c}\text { Hybridity as a process of } \\
\text { technology's 'translation': } \\
\text { customizing a national electronic } \\
\text { patient record }\end{array}$ & 2015 & 5 & 1 \\
\hline 90 & $\begin{array}{l}\text { Fernando, Bernard; } \\
\text { Morrison, Zoe; } \\
\text { et al. }\end{array}$ & $\begin{array}{l}\text { Approaches to recording drug } \\
\text { allergies in electronic health } \\
\text { records: qualitative study }\end{array}$ & 2014 & 5 & 0.83 \\
\hline 91 & $\begin{array}{l}\text { Poulymenopoulou, } \\
\text { M.; Malamateniou, } \\
\text { F.; Vassilacopoulos, } \\
\text { G. }\end{array}$ & $\begin{array}{l}\text { E-EPR: a workflow-based } \\
\text { electronic emergency patient } \\
\text { record }\end{array}$ & 2014 & 5 & 0.83 \\
\hline
\end{tabular}

Table 1b The most cited papers in EHMS between 2008-2018 (Scopus)

\begin{tabular}{|c|c|c|c|c|c|}
\hline Order & Author (s) & Title & $\begin{array}{l}\text { Publication } \\
\text { year }\end{array}$ & $\begin{array}{c}\text { Total } \\
\text { citations }\end{array}$ & $\begin{array}{l}\text { Citation } \\
\text { per year }\end{array}$ \\
\hline 1 & Chang P.H. & $\begin{array}{l}\text { Modeling the management of } \\
\text { electronic health records in } \\
\text { healthcare information systems }\end{array}$ & 2011 & 5 & 0.71 \\
\hline 2 & $\begin{array}{l}\text { Makela K.; Virjo I.; } \\
\text { et al. }\end{array}$ & $\begin{array}{l}\text { Management of electronic patient } \\
\text { record systems in primary } \\
\text { healthcare in a Finnish county. }\end{array}$ & 2010 & 5 & 0.63 \\
\hline 3 & Bar-Lev S. & $\begin{array}{l}\text { The politics of healthcare } \\
\text { informatics: Knowledge } \\
\text { management using an electronic } \\
\text { medical record system }\end{array}$ & 2015 & 4 & 1.0 \\
\hline 4 & $\begin{array}{l}\text { Asghar M.R., } \\
\text { Russello G. }\end{array}$ & $\begin{array}{l}\text { Automating consent management } \\
\text { lifecycle for electronic healthcare } \\
\text { systems }\end{array}$ & 2015 & 2 & 0.5 \\
\hline 5 & $\begin{array}{c}\text { Pascal C.J.; } \\
\text { McInerney C.; et al. }\end{array}$ & $\begin{array}{l}\text { The use of knowledge } \\
\text { management in healthcare: The } \\
\text { implementation of shared care } \\
\text { plans in electronic medical record } \\
\text { systems at one primary care } \\
\text { practice }\end{array}$ & 2013 & 2 & 0.33 \\
\hline
\end{tabular}

Table 2a The most cited documents by EHMS publications (Web of Science)

\begin{tabular}{|c|c|c|c|c|}
\hline Order & Year & Abbreviated reference(s) & $\begin{array}{l}\text { Type of } \\
\text { outlets }\end{array}$ & Citations \\
\hline 1 & 2010 & Sittig, Dean F.; Singh, Hardeep & A & 171 \\
\hline 2 & 2010 & Greenhalgh, Trisha; Hinder, Susan; et al. & A & 134 \\
\hline 3 & 2011 & Lluch, Maria & A & 117 \\
\hline 4 & 2014 & de Lusignan, Simon; Mold, Freda; et al. & A & 73 \\
\hline 5 & 2012 & Estabrooks, Paul A.; Boyle, Maureen; et al. & A & 72 \\
\hline 6 & 2016 & Mandel, Joshua C.; Kreda, David A.; et al. & A & 57 \\
\hline 7 & 2008 & Kazley, Abby S.; Ozcan, Yasar A. & A & 52 \\
\hline
\end{tabular}


Table 2a The most cited documents by EHMS publications (Web of Science) (continued)

\begin{tabular}{|c|c|c|c|c|}
\hline Order & Year & Abbreviated reference(s) & $\begin{array}{l}\text { Type of } \\
\text { outlets }\end{array}$ & Citations \\
\hline 8 & 2012 & Masys, Daniel R.; Jarvik, Gail P.; et al. & $\mathrm{A}$ & 51 \\
\hline 9 & 2008 & Peleg, Mor; Keren, Sagi; Denekamp, Yaron & $\mathrm{A}$ & 51 \\
\hline 10 & 2008 & Terry, Amanda L.; Thorpe, Cathy F.; et al. & $\mathrm{A}$ & 49 \\
\hline 11 & 2012 & Wright, Adam; Poon, Eric G.; et al. & A & 48 \\
\hline 12 & 2010 & Stoves, John; Connolly, John; et al. & A & 45 \\
\hline 13 & 2008 & Wong, Martin C. S.; Jiang, Johnny Y.; et al. & A & 45 \\
\hline 14 & 2013 & Freeman, R.; Moore, L. S. P.; et al. & A & 43 \\
\hline 15 & 2012 & Rothman, Brian; Leonard, Joan C.; Vigoda, Michael M. & A & 38 \\
\hline 16 & 2014 & Ng, Kenney; Ghoting, Amol; et al. & A & 36 \\
\hline 17 & 2012 & Chen, Yu-Yi; Lu, Jun-Chao; Jan, Jinn-Ke & A & 35 \\
\hline 18 & 2009 & Linder, Jeffrey A.; Kaleba, Erin O.; Kmetik, Karen S. & A & 32 \\
\hline 19 & 2014 & Meeks, Derek W.; Takian, Amirhossein; et al. & $\mathrm{A}$ & 31 \\
\hline 20 & 2011 & Wright, Adam; Sittig, Dean F.; et al. & A & 30 \\
\hline 21 & 2012 & Hsu, William; Taira, Ricky K.; et al. & A & 29 \\
\hline 22 & 2014 & Morley, Katherine I.; Wallace, Joshua; et al. & A & 28 \\
\hline 23 & 2012 & Mishra, Abhay Nath; Anderson, Catherine; et al. & A & 27 \\
\hline 24 & 2010 & Harris, Stewart B.; Glazier, Richard H.; et al. & $\mathrm{A}$ & 27 \\
\hline 25 & 2011 & Sittig, Dean F.; Wright, Adam; et al. & A & 26 \\
\hline 26 & 2010 & Cho, InSook; Kim, JeongAh; et al. & A & 25 \\
\hline 27 & 2015 & Pahl, Christina; Zare, Mojtaba; et al. & A & 23 \\
\hline 28 & 2014 & Ancker, Jessica S.; Kern, Lisa M.; et al. & A & 23 \\
\hline 29 & 2013 & Saleem, Jason J.; Flanagan, Mindy E.; et al. & A & 23 \\
\hline 30 & 2012 & McGinn, Carrie Anna; Gagnon, Marie-Pierre; et al. & A & 23 \\
\hline 31 & 2013 & Jarvis, Benjamin; Johnson, Tricia; et al. & A & 22 \\
\hline 32 & 2009 & Sassen, Elizabeth J. & A & 22 \\
\hline 33 & 2012 & O’Reilly, Daria; Holbrook, Anne; et al. & A & 21 \\
\hline 34 & 2016 & Kumar, Rajiv B.; Goren, Nira D.; et al. & A & 20 \\
\hline 35 & 2013 & Wang, Hua-Qiong; Li, Jing-Song; et al. & A & 20 \\
\hline 36 & 2011 & Benhamou, P-Y. & $\mathrm{A}$ & 20 \\
\hline 37 & 2010 & Prociow, Pawel A.; Crowe, John A. & $\mathrm{A}$ & 20 \\
\hline 38 & 2014 & Rahimi, Alireza; Liaw, Siaw-Teng; et al. & $\mathrm{A}$ & 19 \\
\hline 39 & 2012 & Herbek, S.; Eisl, H. A.; et al. & $\mathrm{A}$ & 19 \\
\hline 40 & 2015 & Wright, Adam; McCoy, Allison B.; et al. & $\mathrm{A}$ & 18 \\
\hline 41 & 2014 & Phansalkar, Shobha; Zachariah, Marianne; et al. & A & 18 \\
\hline 42 & 2013 & Kopanitsa, G.; Hildebrand, C.; et al. & A & 18 \\
\hline 43 & 2012 & Banerjee, Dipanjan; Chung, Sukyung; et al. & A & 18 \\
\hline
\end{tabular}


Table 2a The most cited documents by EHMS publications (Web of Science) (continued)

\begin{tabular}{|c|c|c|c|c|}
\hline Order & Year & Abbreviated reference(s) & $\begin{array}{l}\text { Type of } \\
\text { outlets }\end{array}$ & Citations \\
\hline 44 & 2013 & Li, Jing-Song; Zhang, Xiao-Guang; et al. & A & 17 \\
\hline 45 & 2014 & Menon, Shailaja; Smith, Michael W.; et al. & A & 16 \\
\hline 46 & 2013 & Noblin, Alice; Cortelyou-Ward, Kendall; et al. & A & 16 \\
\hline 47 & 2013 & Makam, Anil N.; Nguyen, Oanh K.; et al. & A & 15 \\
\hline 48 & 2011 & Zhang, Jianguo; Zhang, Kai; et al. & A & 15 \\
\hline 49 & 2015 & Ancker, Jessica S.; Kern, Lisa M.; et al. & A & 14 \\
\hline 50 & 2018 & Guo, Rui; Shi, Huixian; et al. & A & 13 \\
\hline 51 & 2018 & Dagher, Gaby G.; Mohler, Jordan; et al. & A & 12 \\
\hline 52 & 2014 & Goldstein, David H.; Phelan, Rachel; et al. & A & 12 \\
\hline 53 & 2012 & Teufel, Ronald J., II; Kazley, Abby Swanson; et al. & A & 12 \\
\hline 54 & 2012 & Li, Jing-Song; Zhang, Xiao-Guang; et al. & A & 12 \\
\hline 55 & 2011 & Newsham, Alexander C.; Johnston, Colin; et al. & A & 12 \\
\hline 56 & 2011 & Xiao, Liang; Cousins, Grainne; et al. & A & 12 \\
\hline 57 & 2010 & Marimon-Sunol, Santiago; Rovira-Barbera, Maria; et al. & A & 11 \\
\hline 58 & 2008 & Grant, C.; Ludbrook, G.; et al. & A & 11 \\
\hline 59 & 2018 & Yadav, Pranjul; Steinbach, Michael; et al. & A & 10 \\
\hline 60 & 2016 & van Velthoven, Michelle Helena; Mastellos, Nikolaos; et al. & A & 10 \\
\hline 61 & 2015 & Liu, Chung-Feng; Cheng, Tain-Junn & A & 10 \\
\hline 62 & 2014 & Shea, Christopher M.; Reiter, Kristin L.; et al. & A & 10 \\
\hline 63 & 2014 & Kontio, Elina; Airola, Antti; et al. & A & 10 \\
\hline 64 & 2017 & Heart, Tsipi; Ben-Assuli, Ofir; Shabtai, Itamar & A & 9 \\
\hline 65 & 2016 & Jawhari, Badeia; Keenan, Louanne; et al. & A & 9 \\
\hline 66 & 2016 & Gagnon, Marie-Pierre; Payne-Gagnon, Julie; et al. & A & 9 \\
\hline 67 & 2014 & McAlearney, Ann Scheck; Hefner, Jennifer L.; et al. & A & 9 \\
\hline 68 & 2013 & Tundia, Namita L.; Kelton, Christina M. L.; et al., & A & 9 \\
\hline 69 & 2011 & Navarro, Ronald A.; Greene, Denise F.; et al. & A & 9 \\
\hline 70 & 2016 & $\begin{array}{l}\text { del Carmen Legaz-Garcia, Maria; Martinez-Costa, Catalina; } \\
\text { et al. }\end{array}$ & A & 8 \\
\hline 71 & 2015 & Sajatovic, Martha; Welter, Elisabeth; et al. & A & 8 \\
\hline 72 & 2015 & Park, Sun Young; Chen, Yunan; Rudkin, Scott & A & 8 \\
\hline 73 & 2016 & $\begin{array}{l}\text { Cardoso de Moraes, Joao Luis; de Souza, Wanderley Lopes; } \\
\text { et al. }\end{array}$ & A & 7 \\
\hline 74 & 2013 & Teufel, Ronald J., II; Kazley, Abby Swanson; et al. & A & 7 \\
\hline 75 & 2010 & Al Mallah, Amr; Guelpa, Paul; et al. & A & 7 \\
\hline 76 & 2018 & Kharrazi, Hadi; Gonzalez, Claudia P.; et al. & A & 6 \\
\hline 77 & 2017 & Plantier, Morgane; Havet, Nathalie; et al. & A & 6 \\
\hline 78 & 2016 & de Ruiter, Hans-Peter; Liaschenko, Joan; Angus, Jan & A & 6 \\
\hline 79 & 2015 & Somolinos, Roberto; Munoz, Adolfo; et al. & A & 6 \\
\hline
\end{tabular}


Table 2a The most cited documents by EHMS publications (Web of Science) (continued)

\begin{tabular}{llccc}
\hline Order & Year & Abbreviated reference(s) & $\begin{array}{c}\text { Type of } \\
\text { outlets }\end{array}$ & Citations \\
\hline 80 & 2015 & McCoy, Allison B.; Wright, Adam; Sittig, Dean F. & A & 6 \\
81 & 2015 & Marceglia, S.; Fontelo, P.; et al. & A & 6 \\
82 & 2014 & Hazlehurst, Brian L.; Lawrence, Jean M.; et al. & A & 6 \\
83 & 2012 & Zaninelli, M.; Campagnoli, A.; et al. & A & 6 \\
84 & 2011 & McEwen, Timothy R.; Elder, Nancy C.; Flach, John M. & A & 6 \\
85 & 2018 & De Pietro, Carlo; Francetic, Igor & A & 5 \\
86 & 2017 & Wang, Shirley V.; Rogers, James R.; et al. & A & 5 \\
87 & 2016 & Matton, Marie-Pier; Toledano, Baruch; et al. & A & 5 \\
88 & 2015 & Kafi, Mohamed Amine; Ben Othman, Jalel; et al. & A & 5 \\
89 & 2015 & Petrakaki, Dimitra; Klecun, Ela & A & 5 \\
90 & 2014 & Fernando, Bernard; Morrison, Zoe; et al. & A & 5 \\
91 & 2014 & Poulymenopoulou, M.; Malamateniou, F.; Vassilacopoulos, G. & A & 5 \\
\hline
\end{tabular}

Note: *Outlets: A - Article; B - conference proceedings, C - Book chapter.

Table 2b The most cited documents by EHMS publications (Scopus)

\begin{tabular}{llccc}
\hline Order & Year & Abbreviated Reference (s) & $\begin{array}{r}\text { Type of } \\
\text { outlets }\end{array}$ & Citations \\
\hline 1 & 2011 & Chang, P.H. & B & 5 \\
2 & 2010 & Makela, K.; Virjo, I.; et al. & A & 5 \\
3 & 2015 & Bar-Lev, S. & A & 4 \\
4 & 2015 & Asghar, M.R., Russello, G. & C & 2 \\
5 & 2013 & Pascal, C.J.; McInerney, C.; et al. & A & 2 \\
\hline
\end{tabular}

Note: Outlets: A - article; B - conference proceedings, $\mathrm{C}$ - book chapter.

In Scopus, the contribution of Chang; Makela, Virjo et al; and Bar-Lev represent the most cited papers in EHMS between the period reviewed. Apart from having the most cited paper in Web of Science on EHMS, Sittig, Dean also had a total of two papers included as part of our review. Wright, Adam has 3 papers in the list being the most productive author in EHMS, while Ancker, Jessica; Li, Jing-Song; and Teufel, Ronald have two papers each.

Table 2 arranged our 96 papers from both Web of Science and Scopus in an order starting from the paper that received the most citation to the least, Table 2 also identifies the type of outlets for these papers in EHMS. In Table 3, we looked into the citation structure for EHMS in Web of Science and Scopus, our work analysed the total number of papers published from 2008 to 2018 and reports the total number of citations they have achieved taken into consideration different citation thresholds. Yearly citation trend from Web of Science reveals that 2010 and 2012 had the highest number of citations in EHMS with a total of 8 and 14 papers respectively. 
Table 3a Yearly citation trend of EHMS (Web of Science)

\begin{tabular}{cccccccccc}
\hline Year & $\begin{array}{c}\text { Total } \\
\text { paper }\end{array}$ & $\begin{array}{c}\text { Total } \\
\text { citation }\end{array}$ & $\geq 200$ & $\geq 100$ & $\geq 50$ & $\geq 20$ & $\geq 10$ & $\geq 5$ & $\geq 1$ \\
\hline 2008 & 5 & 208 & 0 & 0 & 2 & 4 & 5 & 5 & 5 \\
2009 & 2 & 54 & 0 & 0 & 0 & 2 & 2 & 2 & 2 \\
2010 & 8 & 440 & 0 & 2 & 2 & 6 & 7 & 8 & 8 \\
2011 & 9 & 247 & 0 & 1 & 0 & 3 & 7 & 9 & 9 \\
2012 & 14 & 411 & 0 & 0 & 2 & 9 & 13 & 14 & 14 \\
2013 & 10 & 190 & 0 & 0 & 0 & 4 & 8 & 10 & 10 \\
2014 & 15 & 301 & 0 & 0 & 1 & 5 & 11 & 15 & 15 \\
2015 & 11 & 109 & 0 & 0 & 0 & 1 & 4 & 11 & 11 \\
2016 & 9 & 131 & 0 & 0 & 1 & 2 & 3 & 9 & 9 \\
2017 & 3 & 20 & 0 & 0 & 0 & 0 & 0 & 3 & 3 \\
2018 & 5 & 46 & 0 & 0 & 0 & 0 & 3 & 5 & 5 \\
\hline
\end{tabular}

Table 3b Yearly citation trend of EHMS (Scopus)

\begin{tabular}{cccccccccc}
\hline Year & $\begin{array}{c}\text { Total } \\
\text { paper }\end{array}$ & $\begin{array}{c}\text { Total } \\
\text { citation }\end{array}$ & $\geq 200$ & $\geq 100$ & $\geq 50$ & $\geq 20$ & $\geq 10$ & $\geq 5$ & $\geq 1$ \\
\hline 2008 & 0 & 0 & 0 & 0 & 0 & 0 & 0 & 0 & 0 \\
2009 & 0 & 0 & 0 & 0 & 0 & 0 & 0 & 0 & 0 \\
2010 & 1 & 5 & 0 & 0 & 0 & 0 & 0 & 1 & 1 \\
2011 & 1 & 5 & 0 & 0 & 0 & 0 & 0 & 1 & 1 \\
2012 & 0 & 0 & 0 & 0 & 0 & 0 & 0 & 0 & 0 \\
2013 & 1 & 2 & 0 & 0 & 0 & 0 & 0 & 0 & 1 \\
2014 & 0 & 0 & 0 & 0 & 0 & 0 & 0 & 0 & 0 \\
2015 & 2 & 6 & 0 & 0 & 0 & 0 & 0 & 0 & 2 \\
2016 & 0 & 0 & 0 & 0 & 0 & 0 & 0 & 0 & 0 \\
2017 & 0 & 0 & 0 & 0 & 0 & 0 & 0 & 0 & 0 \\
2018 & 0 & 0 & 0 & 0 & 0 & 0 & 0 & 0 & 0 \\
\hline
\end{tabular}

The number of citations and papers in EHMS is declining through time with 2017 seeing the least citations within the period under review. On Scopus, 2015 had the highest number of citations with a total of two papers, followed by 2010 and 2011 who both had five citations each. Our results show that there has been no paper published in EHMS since 2015.

\subsection{Leading authors, institutions and countries in EHMS}

In identifying the most productive and influential authors, institutions and countries in EHMS, we organised our results based on how many papers they have in EHMS from 2008 to 2018 . Note that in the case where there is a tie, we have ranked based on the number of citations. 
Table 4a Authors productivity in EHMS (Web of Science)

\begin{tabular}{|c|c|c|c|c|c|c|c|c|c|c|}
\hline Order & $A N$ & Institution & $C T$ & $T P$ & $T C$ & h-index & $A C$ & $\geq 200$ & $\geq 100$ & $\geq 50$ \\
\hline 1 & $\begin{array}{l}\text { Wright, } \\
\text { Adam }\end{array}$ & $\begin{array}{l}\text { Harvard Medical } \\
\text { School }\end{array}$ & USA & 3 & 96 & 3 & 33.67 & 0 & 0 & 1 \\
\hline 2 & $\begin{array}{l}\text { Sittig, Dean } \\
\text { F. }\end{array}$ & $\begin{array}{l}\text { University of } \\
\text { Texas }\end{array}$ & USA & 2 & 197 & 2 & 105 & 0 & 1 & 1 \\
\hline 3 & $\begin{array}{l}\text { Ancker, } \\
\text { Jessica S. }\end{array}$ & Cornell University & USA & 2 & 37 & 2 & 19.5 & 0 & 0 & 0 \\
\hline 4 & $\begin{array}{c}\mathrm{Li}, \\
\text { Jing-Song }\end{array}$ & $\begin{array}{l}\text { Zhejiang } \\
\text { University }\end{array}$ & China & 2 & 29 & 2 & 14.5 & 0 & 0 & 0 \\
\hline 5 & $\begin{array}{l}\text { Teufel, } \\
\text { Ronald J., II }\end{array}$ & $\begin{array}{l}\text { Medical University } \\
\text { of South Carolina }\end{array}$ & USA & 2 & 19 & 2 & 10.5 & 0 & 0 & 0 \\
\hline 6 & $\begin{array}{c}\text { Greenhalgh, } \\
\text { Trisha }\end{array}$ & $\begin{array}{l}\text { London School of } \\
\text { Medicine and } \\
\text { Dentistry }\end{array}$ & UK & 1 & 134 & 1 & 134 & 0 & 1 & 1 \\
\hline 7 & $\begin{array}{l}\text { Lluch, } \\
\text { Maria }\end{array}$ & $\begin{array}{l}\text { London School of } \\
\text { Economics and } \\
\text { Political Science }\end{array}$ & UK & 1 & 117 & 1 & 117 & 0 & 1 & 1 \\
\hline 8 & $\begin{array}{l}\text { de } \\
\text { Lusignan, } \\
\text { Simon }\end{array}$ & $\begin{array}{l}\text { University of } \\
\text { Surrey }\end{array}$ & UK & 1 & 73 & 1 & 73 & 0 & 0 & 1 \\
\hline 9 & $\begin{array}{l}\text { Estabrooks, } \\
\text { Paul A. }\end{array}$ & $\begin{array}{c}\text { Virginia Tech, } \\
\text { USA }\end{array}$ & USA & 1 & 72 & 1 & 72 & 0 & 0 & 1 \\
\hline 10 & $\begin{array}{l}\text { Mandel, } \\
\text { Joshua C. }\end{array}$ & $\begin{array}{l}\text { Harvard Medical } \\
\text { School, Boston, } \\
\text { MA, USA }\end{array}$ & USA & 1 & 57 & 1 & 57 & 0 & 0 & 1 \\
\hline 11 & $\begin{array}{l}\text { Kazley, } \\
\text { Abby S. }\end{array}$ & $\begin{array}{l}\text { Medical University } \\
\text { of South Carolina }\end{array}$ & USA & 1 & 52 & 1 & 52 & 0 & 0 & 1 \\
\hline 12 & $\begin{array}{l}\text { Masys, } \\
\text { Daniel R. }\end{array}$ & $\begin{array}{l}\text { University of } \\
\text { Washington }\end{array}$ & USA & 1 & 51 & 1 & 51 & 0 & 0 & 1 \\
\hline 13 & Peleg, Mor & University of Haifa & Israel & 1 & 51 & 1 & 51 & 0 & 0 & 1 \\
\hline 14 & $\begin{array}{c}\text { Terry, } \\
\text { Amanda L. }\end{array}$ & $\begin{array}{l}\text { The University of } \\
\text { Western Ontario }\end{array}$ & Canada & 1 & 49 & 1 & 49 & 0 & 0 & 0 \\
\hline 15 & $\begin{array}{l}\text { Stoves, } \\
\text { John }\end{array}$ & $\begin{array}{l}\text { NHS Foundation } \\
\text { Trust, UK }\end{array}$ & UK & 1 & 45 & 1 & 45 & 0 & 0 & 0 \\
\hline 16 & $\begin{array}{l}\text { Wong, } \\
\text { Martin C. S. }\end{array}$ & $\begin{array}{c}\text { Chinese University } \\
\text { of Hong Kong }\end{array}$ & Hong Kong & 1 & 45 & 1 & 45 & 0 & 0 & 0 \\
\hline 17 & Freeman, R. & $\begin{array}{l}\text { Imperial College, } \\
\text { London, UK }\end{array}$ & UK & 1 & 43 & 1 & 43 & 0 & 0 & 0 \\
\hline 18 & $\begin{array}{l}\text { Rothman, } \\
\text { Brian }\end{array}$ & $\begin{array}{c}\text { Vanderbilt } \\
\text { University, USA }\end{array}$ & USA & 1 & 38 & 1 & 38 & 0 & 0 & 0 \\
\hline 19 & Ng, Kenney & $\begin{array}{l}\text { IBM TJ Watson } \\
\text { Research Center }\end{array}$ & USA & 1 & 36 & 1 & 36 & 0 & 0 & 0 \\
\hline 20 & $\begin{array}{l}\text { Chen, } \\
\text { Yu-Yi }\end{array}$ & $\begin{array}{l}\text { National Chung } \\
\text { Hsing University }\end{array}$ & China & 1 & 35 & 1 & 35 & 0 & 0 & 0 \\
\hline
\end{tabular}

Note: Abbreviations: author name - AN; country - CT; total paper - TP; total citation $\mathrm{TC}$; average citation per item - AC. 
Table 4a Authors productivity in EHMS (Web of Science) (continued)

\begin{tabular}{|c|c|c|c|c|c|c|c|c|c|c|}
\hline Order & $A N$ & Institution & $C T$ & $T P$ & $T C$ & h-index & $A C$ & $\geq 200$ & $\geq 100$ & $\geq 50$ \\
\hline 21 & $\begin{array}{l}\text { Linder, } \\
\text { Jeffrey A. }\end{array}$ & $\begin{array}{l}\text { Harvard Medical } \\
\text { School }\end{array}$ & USA & 1 & 32 & 1 & 32 & 0 & 0 & 0 \\
\hline 22 & $\begin{array}{l}\text { Meeks, } \\
\text { Derek W. }\end{array}$ & $\begin{array}{l}\text { Baylor College of } \\
\text { Medicine }\end{array}$ & USA & 1 & 31 & 1 & 31 & 0 & 0 & 0 \\
\hline 23 & $\begin{array}{c}\text { Hsu, } \\
\text { William }\end{array}$ & $\begin{array}{c}\text { University of } \\
\text { California at Los } \\
\text { Angeles }\end{array}$ & USA & 1 & 29 & 1 & 29 & 0 & 0 & 0 \\
\hline 24 & $\begin{array}{l}\text { Morley, } \\
\text { Katherine I. }\end{array}$ & $\begin{array}{l}\text { University College } \\
\text { London }\end{array}$ & UK & 1 & 28 & 1 & 28 & 0 & 0 & 0 \\
\hline 25 & $\begin{array}{l}\text { Mishra, } \\
\text { Abhay Nath }\end{array}$ & $\begin{array}{c}\text { Georgia State } \\
\text { University }\end{array}$ & USA & 1 & 27 & 1 & 27 & 0 & 0 & 0 \\
\hline 26 & $\begin{array}{l}\text { Harris, } \\
\text { Stewart B. }\end{array}$ & $\begin{array}{l}\text { The University of } \\
\text { Western Ontario }\end{array}$ & Canada & 1 & 27 & 1 & 27 & 0 & 0 & 0 \\
\hline 27 & $\begin{array}{l}\text { Cho, } \\
\text { InSook }\end{array}$ & Inha University & $\begin{array}{l}\text { South } \\
\text { Korea }\end{array}$ & 1 & 25 & 1 & 25 & 0 & 0 & 0 \\
\hline 28 & $\begin{array}{l}\text { Pahl, } \\
\text { Christina }\end{array}$ & $\begin{array}{l}\text { Ilmenau University } \\
\text { of Technology }\end{array}$ & Germany & 1 & 23 & 1 & 23 & 0 & 0 & 0 \\
\hline 29 & $\begin{array}{l}\text { Saleem, } \\
\text { Jason J. }\end{array}$ & Indiana University & USA & 1 & 23 & 1 & 23 & 0 & 0 & 0 \\
\hline 30 & $\begin{array}{l}\text { McGinn, } \\
\text { Carrie Anna }\end{array}$ & $\begin{array}{l}\text { Institut de } \\
\text { réadaptation en } \\
\text { déficience } \\
\text { physique de } \\
\text { Québec }\end{array}$ & Canada & 1 & 23 & 1 & 23 & 0 & 0 & 0 \\
\hline 31 & $\begin{array}{l}\text { Jarvis, } \\
\text { Benjamin }\end{array}$ & $\begin{array}{l}\text { NorthShore } \\
\text { University }\end{array}$ & USA & 1 & 22 & 1 & 22 & 0 & 0 & 0 \\
\hline 32 & $\begin{array}{l}\text { Sassen, } \\
\text { Elizabeth J. }\end{array}$ & Loyola University & USA & 1 & 22 & 1 & 22 & 0 & 0 & 0 \\
\hline 33 & $\begin{array}{l}\text { O'Reilly, } \\
\text { Daria }\end{array}$ & $\begin{array}{l}\text { McMaster } \\
\text { University }\end{array}$ & Canada & 1 & 21 & 1 & 21 & 0 & 0 & 0 \\
\hline 34 & $\begin{array}{l}\text { Kumar, } \\
\text { Rajiv B. }\end{array}$ & $\begin{array}{l}\text { Stanford } \\
\text { University }\end{array}$ & USA & 1 & 20 & 1 & 20 & 0 & 0 & 0 \\
\hline 35 & $\begin{array}{c}\text { Wang, } \\
\text { Hua-Qiong }\end{array}$ & $\begin{array}{l}\text { Zhejiang } \\
\text { University }\end{array}$ & China & 1 & 20 & 1 & 20 & 0 & 0 & 0 \\
\hline 36 & $\begin{array}{l}\text { Benhamou, } \\
\text { P-Y. }\end{array}$ & $\begin{array}{c}\text { Joseph Fourier } \\
\text { University, } \\
\text { Grenoble, France }\end{array}$ & France & 1 & 20 & 1 & 20 & 0 & 0 & 0 \\
\hline 37 & $\begin{array}{l}\text { Prociow, } \\
\text { Pawel A. }\end{array}$ & $\begin{array}{l}\text { University of } \\
\text { Nottingham, } \\
\text { Nottingham, UK }\end{array}$ & UK & 1 & 20 & 1 & 20 & 0 & 0 & 0 \\
\hline 38 & $\begin{array}{l}\text { Rahimi, } \\
\text { Alireza }\end{array}$ & UNSW, Australia & Australia & 1 & 19 & 1 & 19 & 0 & 0 & 0 \\
\hline 39 & Herbek, S. & $\begin{array}{c}\text { ELGA GmbH } \\
\text { Vienna, Austria }\end{array}$ & Austria & 1 & 19 & 1 & 19 & 0 & 0 & 0 \\
\hline
\end{tabular}

Note: Abbreviations: author name - AN; country - CT; total paper - TP; total citation $\mathrm{TC}$; average citation per item - AC. 
Table 4a Authors productivity in EHMS (Web of Science) (continued)

\begin{tabular}{|c|c|c|c|c|c|c|c|c|c|c|}
\hline Order & $A N$ & Institution & $C T$ & $T P$ & $T C$ & h-index & $A C$ & $\geq 200$ & $\geq 100$ & $\geq 50$ \\
\hline 40 & $\begin{array}{l}\text { Phansalkar, } \\
\text { Shobha }\end{array}$ & $\begin{array}{l}\text { Partners } \\
\text { Healthcare } \\
\text { Systems }\end{array}$ & USA & 1 & 18 & 1 & 18 & 0 & 0 & 0 \\
\hline 41 & $\begin{array}{l}\text { Kopanitsa, } \\
\text { G. }\end{array}$ & $\begin{array}{c}\text { German Research } \\
\text { Center for } \\
\text { Environmental } \\
\text { Health, } \\
\text { Neuherberg, } \\
\text { Germany }\end{array}$ & Germany & 1 & 18 & 1 & 18 & 0 & 0 & 0 \\
\hline 42 & $\begin{array}{l}\text { Banerjee, } \\
\text { Dipanjan }\end{array}$ & $\begin{array}{l}\text { Stanford } \\
\text { University }\end{array}$ & USA & 1 & 18 & 1 & 18 & 0 & 0 & 0 \\
\hline 43 & $\begin{array}{l}\text { Menon, } \\
\text { Shailaja }\end{array}$ & $\begin{array}{l}\text { Baylor College of } \\
\text { Medicine }\end{array}$ & USA & 1 & 16 & 1 & 16 & 0 & 0 & 0 \\
\hline 44 & $\begin{array}{l}\text { Noblin, } \\
\text { Alice }\end{array}$ & $\begin{array}{l}\text { University of } \\
\text { Central Florida }\end{array}$ & USA & 1 & 16 & 1 & 16 & 0 & 0 & 0 \\
\hline 45 & $\begin{array}{l}\text { Makam, } \\
\text { Anil N. }\end{array}$ & $\begin{array}{l}\text { University of } \\
\text { California San } \\
\text { Francisco }\end{array}$ & USA & 1 & 15 & 1 & 15 & 0 & 0 & 0 \\
\hline 46 & $\begin{array}{l}\text { Zhang, } \\
\text { Jianguo }\end{array}$ & $\begin{array}{c}\text { Shanghai Institute } \\
\text { of Technical } \\
\text { Physics }\end{array}$ & China & 1 & 15 & 1 & 15 & 0 & 0 & 0 \\
\hline 47 & Guo, Rui & Xi'an University & China & 1 & 13 & 1 & 13 & 0 & 0 & 0 \\
\hline 48 & $\begin{array}{l}\text { Dagher, } \\
\text { Gaby G. }\end{array}$ & $\begin{array}{l}\text { Boise State } \\
\text { University }\end{array}$ & USA & 1 & 12 & 1 & 12 & 0 & 0 & 0 \\
\hline 49 & $\begin{array}{l}\text { Goldstein, } \\
\text { David H. }\end{array}$ & $\begin{array}{l}\text { Queen's } \\
\text { University }\end{array}$ & Canada & 1 & 12 & 1 & 12 & 0 & 0 & 0 \\
\hline 50 & $\begin{array}{l}\text { Newsham, } \\
\text { Alexander } \\
\text { C. }\end{array}$ & $\begin{array}{l}\text { University of } \\
\text { Leeds }\end{array}$ & UK & 1 & 12 & 1 & 12 & 0 & 0 & 0 \\
\hline 51 & Xiao, Liang & $\begin{array}{l}\text { Royal College of } \\
\text { Surgeons in } \\
\text { Ireland }\end{array}$ & Ireland & 1 & 12 & 1 & 12 & 0 & 0 & 0 \\
\hline 52 & $\begin{array}{l}\text { Marimon- } \\
\text { Sunol, } \\
\text { Santiago }\end{array}$ & $\begin{array}{l}\text { epartament de } \\
\text { Salut, Generalitat } \\
\text { de Catalunya, } \\
\text { Barcelona, España }\end{array}$ & Spain & 1 & 11 & 1 & 11 & 0 & 0 & 0 \\
\hline 53 & Grant, C. & $\begin{array}{l}\text { University of } \\
\text { Adelaide }\end{array}$ & Australia & 1 & 11 & 1 & 11 & 0 & 0 & 0 \\
\hline 54 & $\begin{array}{l}\text { Yadav, } \\
\text { Pranjul }\end{array}$ & $\begin{array}{c}\text { University of } \\
\text { Minnesota - Twin } \\
\text { Cities }\end{array}$ & USA & 1 & 10 & 1 & 10 & 0 & 0 & 0 \\
\hline 55 & $\begin{array}{l}\text { van } \\
\text { Velthoven, } \\
\text { Michelle } \\
\text { Helena }\end{array}$ & $\begin{array}{l}\text { Imperial College } \\
\text { London }\end{array}$ & UK & 1 & 10 & 1 & 10 & 0 & 0 & 0 \\
\hline
\end{tabular}

Note: Abbreviations: author name - AN; country - CT; total paper - TP; total citation TC; average citation per item - AC. 
Table 4a Authors productivity in EHMS (Web of Science) (continued)

\begin{tabular}{|c|c|c|c|c|c|c|c|c|c|c|}
\hline Order & $A N$ & Institution & $C T$ & $T P$ & $T C$ & h-index & $A C$ & $\geq 200$ & $\geq 100$ & $\geq 50$ \\
\hline 56 & $\begin{array}{l}\text { Liu, } \\
\text { Chung- } \\
\text { Feng }\end{array}$ & $\begin{array}{c}\text { Chia Nan } \\
\text { University of } \\
\text { Pharmacy and } \\
\text { Science }\end{array}$ & Taiwan & 1 & 10 & 1 & 10 & 0 & 0 & 0 \\
\hline 57 & $\begin{array}{l}\text { Shea, } \\
\text { Christopher } \\
\text { M. }\end{array}$ & $\begin{array}{l}\text { University of } \\
\text { North Carolina- } \\
\text { Chapel Hill }\end{array}$ & USA & 1 & 10 & 1 & 10 & 0 & 0 & 0 \\
\hline 58 & $\begin{array}{c}\text { Kontio, } \\
\text { Elina }\end{array}$ & $\begin{array}{l}\text { University of } \\
\text { Turku }\end{array}$ & Finland & 1 & 10 & 1 & 10 & 0 & 0 & 0 \\
\hline 59 & Heart, Tsipi & $\begin{array}{l}\text { Ono Academic } \\
\text { College }\end{array}$ & Israel & 1 & 9 & 1 & 9 & 0 & 0 & 0 \\
\hline 60 & $\begin{array}{l}\text { Jawhari, } \\
\text { Badeia }\end{array}$ & $\begin{array}{l}\text { University of } \\
\text { Alberta }\end{array}$ & Canada & 1 & 9 & 1 & 9 & 0 & 0 & 0 \\
\hline 61 & $\begin{array}{l}\text { Gagnon, } \\
\text { Marie- } \\
\text { Pierre }\end{array}$ & Université Laval & Canada & 1 & 9 & 1 & 9 & 0 & 0 & 0 \\
\hline 62 & $\begin{array}{l}\text { McAlearne } \\
\text { y, Ann } \\
\text { Scheck }\end{array}$ & $\begin{array}{l}\text { Ohio State } \\
\text { University }\end{array}$ & USA & 1 & 9 & 1 & 9 & 0 & 0 & 0 \\
\hline 63 & $\begin{array}{l}\text { Tundia, } \\
\text { Namita L. }\end{array}$ & $\begin{array}{l}\text { University of } \\
\text { Cincinnati }\end{array}$ & USA & 1 & 9 & 1 & 9 & 0 & 0 & 0 \\
\hline 64 & $\begin{array}{l}\text { Navarro, } \\
\text { Ronald A. }\end{array}$ & $\begin{array}{c}\text { South Bay Medical } \\
\text { Center }\end{array}$ & USA & 1 & 9 & 1 & 9 & 0 & 0 & 0 \\
\hline 65 & $\begin{array}{l}\text { del Carmen } \\
\text { Legaz- } \\
\text { Garcia, } \\
\text { Maria }\end{array}$ & $\begin{array}{l}\text { Universidad de } \\
\text { Murcia, Spain }\end{array}$ & Spain & 1 & 8 & 1 & 8 & 0 & 0 & 0 \\
\hline 66 & $\begin{array}{l}\text { Sajatovic, } \\
\text { Martha }\end{array}$ & $\begin{array}{c}\text { Case Western } \\
\text { Reserve University }\end{array}$ & USA & 1 & 8 & 1 & 8 & 0 & 0 & 0 \\
\hline 67 & $\begin{array}{l}\text { Park, Sun } \\
\text { Young }\end{array}$ & $\begin{array}{c}\text { University of } \\
\text { California, Irvine }\end{array}$ & USA & 1 & 8 & 1 & 8 & 0 & 0 & 0 \\
\hline 68 & $\begin{array}{l}\text { Cardoso de } \\
\text { Moraes, } \\
\text { Joao Luis }\end{array}$ & $\begin{array}{l}\text { Federal University } \\
\text { of São Carlos }\end{array}$ & Brazil & 1 & 7 & 1 & 7 & 0 & 0 & 0 \\
\hline 69 & $\begin{array}{l}\text { Al Mallah, } \\
\text { Amr }\end{array}$ & $\begin{array}{l}\text { Montreal Heart } \\
\text { Institute } \\
\text { Pharmacogenomic } \\
\text { s Centre; }\end{array}$ & Canada & 1 & 7 & 1 & 7 & 0 & 0 & 0 \\
\hline 70 & $\begin{array}{l}\text { Kharrazi, } \\
\text { Hadi }\end{array}$ & $\begin{array}{l}\text { Johns Hopkins } \\
\text { Bloomberg School } \\
\text { of Public Health }\end{array}$ & USA & 1 & 6 & 1 & 6 & 0 & 0 & 0 \\
\hline 71 & $\begin{array}{l}\text { Plantier, } \\
\text { Morgane }\end{array}$ & $\begin{array}{l}\text { Centre de lutte } \\
\text { contre le cancer } \\
\text { Léon Bérard }\end{array}$ & France & 1 & 6 & 1 & 6 & 0 & 0 & 0 \\
\hline
\end{tabular}

Note: Abbreviations: author name - AN; country - CT; total paper - TP; total citation TC; average citation per item - AC. 
Table 4a Authors productivity in EHMS (Web of Science) (continued)

\begin{tabular}{|c|c|c|c|c|c|c|c|c|c|c|}
\hline Order & $A N$ & Institution & $C T$ & $T P$ & $T C$ & h-index & $A C$ & $\geq 200$ & $\geq 100$ & $\geq 50$ \\
\hline 72 & $\begin{array}{l}\text { de Ruiter, } \\
\text { Hans-Peter }\end{array}$ & $\begin{array}{l}\text { Minnesota State } \\
\text { University }\end{array}$ & USA & 1 & 6 & 1 & 6 & 0 & 0 & 0 \\
\hline 73 & $\begin{array}{l}\text { Somolinos, } \\
\text { Roberto }\end{array}$ & $\begin{array}{c}\text { University } \\
\text { Hospital Puerta de } \\
\text { Hierro } \\
\text { Majadahonda }\end{array}$ & Spain & 1 & 6 & 1 & 6 & 0 & 0 & 0 \\
\hline 74 & $\begin{array}{l}\text { McCoy, } \\
\text { Allison B. }\end{array}$ & $\begin{array}{c}\text { Tulane University } \\
\text { School of Public } \\
\text { Health and } \\
\text { Tropical Medicine }\end{array}$ & USA & 1 & 6 & 1 & 6 & 0 & 0 & 0 \\
\hline 75 & $\begin{array}{l}\text { Marceglia, } \\
\text { S. }\end{array}$ & $\begin{array}{l}\text { U.S. National } \\
\text { Library of } \\
\text { Medicine }\end{array}$ & USA & 1 & 6 & 1 & 6 & 0 & 0 & 0 \\
\hline 76 & $\begin{array}{l}\text { Hazlehurst, } \\
\text { Brian L. }\end{array}$ & $\begin{array}{l}\text { Kaiser Permanente } \\
\text { Northwest Center } \\
\text { for Health } \\
\text { Research }\end{array}$ & USA & 1 & 6 & 1 & 6 & 0 & 0 & 0 \\
\hline 77 & $\begin{array}{l}\text { Zaninelli, } \\
\text { M. }\end{array}$ & $\begin{array}{c}\text { Università } \\
\text { Telematica San } \\
\text { Raffaele Roma }\end{array}$ & Italy & 1 & 6 & 1 & 6 & 0 & 0 & 0 \\
\hline 78 & $\begin{array}{l}\text { McEwen, } \\
\text { Timothy R. }\end{array}$ & $\begin{array}{l}\text { Wright State } \\
\text { University }\end{array}$ & USA & 1 & 6 & 1 & 6 & 0 & 0 & 0 \\
\hline 79 & $\begin{array}{l}\text { De Pietro, } \\
\text { Carlo }\end{array}$ & $\begin{array}{l}\text { University of } \\
\text { Applied Sciences } \\
\text { and Arts of } \\
\text { Southern } \\
\text { Switzerland }\end{array}$ & Switzerland & 1 & 5 & 1 & 5 & 0 & 0 & 0 \\
\hline 80 & $\begin{array}{c}\text { Wang, } \\
\text { Shirley V. }\end{array}$ & $\begin{array}{c}\text { Harvard Medical } \\
\text { School, Boston, } \\
\text { MA, USA }\end{array}$ & USA & 1 & 5 & 1 & 5 & 0 & 0 & 0 \\
\hline 81 & $\begin{array}{l}\text { Matton, } \\
\text { Marie-Pier }\end{array}$ & $\begin{array}{c}\text { Sainte-Justine } \\
\text { Hospital, } \\
\text { Montreal, Québec }\end{array}$ & Canada & 1 & 5 & 1 & 5 & 0 & 0 & 0 \\
\hline 82 & $\begin{array}{l}\text { Kafi, } \\
\text { Mohamed } \\
\text { Amine }\end{array}$ & $\begin{array}{l}\text { University of } \\
\text { Science and } \\
\text { Technology } \\
\text { Houari }\end{array}$ & Algeria & 1 & 5 & 1 & 5 & 0 & 0 & 0 \\
\hline 83 & $\begin{array}{l}\text { Petrakaki, } \\
\text { Dimitra }\end{array}$ & $\begin{array}{l}\text { University of } \\
\text { Sussex }\end{array}$ & UK & 1 & 5 & 1 & 5 & 0 & 0 & 0 \\
\hline 84 & $\begin{array}{l}\text { Fernando, } \\
\text { Bernard }\end{array}$ & $\begin{array}{l}\text { University of } \\
\text { Edinburgh }\end{array}$ & UK & 1 & 5 & 1 & 5 & 0 & 0 & 0 \\
\hline 85 & $\begin{array}{l}\text { Poulymeno } \\
\text { poulou, M. }\end{array}$ & $\begin{array}{c}\text { University of } \\
\text { Piraeus, Greece }\end{array}$ & Greece & 1 & 5 & 1 & 5 & 0 & 0 & 0 \\
\hline
\end{tabular}

Note: Abbreviations: author name - AN; country - CT; total paper - TP; total citation $\mathrm{TC}$; average citation per item - AC. 
Table 4b Authors productivity in EHMS (Scopus)

\begin{tabular}{|c|c|c|c|c|c|c|c|c|c|c|}
\hline Order & $A N$ & Institution & $C T$ & $T P$ & $T C$ & $h$-index & $A C$ & $\geq 200$ & $\geq 100$ & $\geq 50$ \\
\hline 1 & $\begin{array}{l}\text { Chang, } \\
\text { P.H. }\end{array}$ & $\begin{array}{l}\text { Lawrence } \\
\text { Technological } \\
\text { University }\end{array}$ & USA & 1 & 5 & 1 & 5 & 0 & 0 & 0 \\
\hline 2 & Makela, K. & $\begin{array}{l}\text { Tampere University } \\
\text { of Technology }\end{array}$ & Finland & 1 & 5 & 1 & 5 & 0 & 0 & 0 \\
\hline 3 & Bar-Lev, S. & $\begin{array}{l}\text { Ruppin Academic } \\
\text { Centre }\end{array}$ & Israel & 1 & 4 & 1 & 4 & 0 & 0 & 0 \\
\hline 4 & $\begin{array}{l}\text { Asghar, } \\
\text { M.R. }\end{array}$ & $\begin{array}{l}\text { University of } \\
\text { Auckland }\end{array}$ & $\begin{array}{c}\text { New } \\
\text { Zealand }\end{array}$ & 1 & 2 & 1 & 2 & 0 & 0 & 0 \\
\hline 5 & Pascal, C.J. & Rutgers University & USA & 1 & 2 & 1 & 2 & 0 & 0 & 0 \\
\hline
\end{tabular}

Note: Abbreviations: author name - AN; country - CT; total paper - TP; total citation $\mathrm{TC}$; average citation per item - AC.

Table 4c Most productive institutions in EHMS (Web of Science)

\begin{tabular}{|c|c|c|c|}
\hline Order & Institution & $\begin{array}{c}\text { Total } \\
\text { paper }\end{array}$ & $\begin{array}{c}\text { Total } \\
\text { citation }\end{array}$ \\
\hline 1 & Harvard Medical School & 6 & 190 \\
\hline 2 & Zhejiang University & 3 & 49 \\
\hline 3 & Medical University of South Carolina & 3 & 71 \\
\hline 4 & University of Texas & 2 & 197 \\
\hline 5 & Cornell University & 2 & 37 \\
\hline 6 & The University of Western Ontario & 2 & 76 \\
\hline 7 & Imperial College London & 2 & 53 \\
\hline 8 & Baylor College of Medicine & 2 & 47 \\
\hline 9 & Stanford University & 2 & 38 \\
\hline 10 & London School of Medicine and Dentistry & 1 & 134 \\
\hline 11 & London School of Economics and Political Science & 1 & 117 \\
\hline 12 & University of Surrey & 1 & 73 \\
\hline 13 & Virginia Tech, USA & 1 & 72 \\
\hline 14 & University of Washington & 1 & 51 \\
\hline 15 & University of Haifa & 1 & 51 \\
\hline 16 & NHS Foundation Trust, UK & 1 & 45 \\
\hline 17 & Chinese University of Hong Kong & 1 & 45 \\
\hline 18 & Vanderbilt University, USA & 1 & 38 \\
\hline 19 & IBM TJ Watson Research Center & 1 & 36 \\
\hline 20 & National Chung Hsing University & 1 & 35 \\
\hline 21 & University of California at Los Angeles & 1 & 29 \\
\hline 22 & University College London & 1 & 28 \\
\hline 23 & Georgia State University & 1 & 27 \\
\hline 24 & Inha University & 1 & 25 \\
\hline 25 & Ilmenau University of Technology & 1 & 23 \\
\hline
\end{tabular}


Table 4c Most productive institutions in EHMS (Web of Science) (continued)

\begin{tabular}{|c|c|c|c|}
\hline Order & Institution & $\begin{array}{l}\text { Total } \\
\text { paper }\end{array}$ & $\begin{array}{c}\text { Total } \\
\text { citation }\end{array}$ \\
\hline 26 & Indiana University & 1 & 23 \\
\hline 27 & Institut de réadaptation en déficience physique de Québec & 1 & 23 \\
\hline 28 & NorthShore University & 1 & 22 \\
\hline 29 & Loyola University & 1 & 22 \\
\hline 30 & McMaster University & 1 & 21 \\
\hline 31 & Joseph Fourier University, Grenoble, France & 1 & 20 \\
\hline 32 & University of Nottingham, Nottingham, UK & 1 & 20 \\
\hline 33 & UNSW, Australia & 1 & 19 \\
\hline 34 & ELGA GmbH Vienna, Austria & 1 & 19 \\
\hline 35 & Partners Healthcare Systems & 1 & 18 \\
\hline 36 & $\begin{array}{l}\text { German Research Center for Environmental Health, } \\
\text { Neuherberg, Germany }\end{array}$ & 1 & 18 \\
\hline 37 & University of Central Florida & 1 & 16 \\
\hline 38 & University of California San Francisco & 1 & 15 \\
\hline 39 & Shanghai Institute of Technical Physics & 1 & 15 \\
\hline 40 & Xi'an University & 1 & 13 \\
\hline 41 & Boise State University & 1 & 12 \\
\hline 42 & Queen's University & 1 & 12 \\
\hline 43 & University of Leeds & 1 & 12 \\
\hline 44 & Royal College of Surgeons in Ireland & 1 & 12 \\
\hline 45 & $\begin{array}{c}\text { Departament de Salut, Generalitat de Catalunya, Barcelona, } \\
\text { España }\end{array}$ & 1 & 11 \\
\hline 46 & University of Adelaide & 1 & 11 \\
\hline 47 & University of Minnesota - Twin Cities & 1 & 10 \\
\hline 48 & Chia Nan University of Pharmacy and Science & 1 & 10 \\
\hline 49 & University of North Carolina-Chapel Hill & 1 & 10 \\
\hline 50 & University of Turku & 1 & 10 \\
\hline 51 & Ono Academic College & 1 & 9 \\
\hline 52 & University of Alberta & 1 & 9 \\
\hline 53 & Université Laval & 1 & 9 \\
\hline 54 & Ohio State University & 1 & 9 \\
\hline 55 & University of Cincinnati & 1 & 9 \\
\hline 56 & South Bay Medical Center & 1 & 9 \\
\hline 57 & Universidad de Murcia, Spain & 1 & 8 \\
\hline 58 & Case Western Reserve University & 1 & 8 \\
\hline 59 & University of California, Irvine & 1 & 8 \\
\hline 60 & Federal University of São Carlos & 1 & 7 \\
\hline 61 & Montreal Heart Institute Pharmacogenomics Centre & 1 & 7 \\
\hline
\end{tabular}


Table 4c Most productive institutions in EHMS (Web of Science) (continued)

\begin{tabular}{lccc}
\hline Order & Institution & $\begin{array}{c}\text { Total } \\
\text { paper }\end{array}$ & $\begin{array}{c}\text { Total } \\
\text { citation }\end{array}$ \\
\hline 62 & Johns Hopkins Bloomberg School of Public Health & 1 & 6 \\
63 & Centre de lutte contre le cancer Léon Bérard & 1 & 6 \\
64 & Minnesota State University & 1 & 6 \\
65 & University Hospital Puerta de Hierro Majadahonda & 1 & 6 \\
66 & Tulane University School of Public Health and Tropical & 1 & 6 \\
67 & Uedicine & 1 & 6 \\
68 & Uaiser Permanente Northwest Center for Health Research & 1 & 6 \\
69 & Università Telematica San Raffaele Roma & 1 & 6 \\
70 & Wright State University & 1 & 6 \\
71 & University of Applied Sciences and Arts of Southern & 1 & 5 \\
75 & Switzerland & & 5 \\
73 & Sainte-Justine Hospital, Montreal, Québec & 1 & 5 \\
\hline 76 & University of Science and Technology Houari & 1 & 5
\end{tabular}

Table 4d Most productive institutions in EHMS (Scopus)

\begin{tabular}{lccc}
\hline Order & Institution & $\begin{array}{c}\text { Total } \\
\text { paper }\end{array}$ & $\begin{array}{c}\text { Total } \\
\text { citation }\end{array}$ \\
\hline 1 & Lawrence Technological University & 1 & 5 \\
2 & Tampere University of Technology & 1 & 5 \\
3 & Ruppin Academic Centre & 1 & 4 \\
4 & University of Auckland & 1 & 2 \\
5 & Rutgers University & 1 & 2 \\
\hline
\end{tabular}

In Web of Science, Wright, Adam has three papers in the list being the most productive author in EHMS, while Sittig, Dean; Ancker, Jessica; Li, Jing-Song and Teufel, Ronald have two papers each. We observed that the first three most productive authors are affiliated to institutions in the USA. All authors shown in our result on Scopus have one paper each; hence, Chang and Makela have been ranked as the most productive authors in Scopus in EHMS within the period we have reviewed. Table $4 \mathrm{a}$ and $4 \mathrm{~b}$ presents this result in detail for all the 96 papers included as part of our review.

Tables $4 \mathrm{c}$ and $4 \mathrm{~d}$ show the most productive institutions in EHMS, Harvard medical school leads the table in Web of Science; interestingly, Wright, Adam who is the most productive author in Web of Science from our result and analysis is affiliated to this institution. Zhejiang University and Medical University of South Carolina took the second and third places; further investigation reveals that these three institutions have dedicated more resources to medical research in their respective regions and have leaped to top rank among other institutions in terms of medical research funding and output. 
All institutions identified in Scopus have same number of papers; hence, we have ranked based on the number of citations; Lawrence Technological University and Tampere University of Technology are the most productive institutions on Scopus with one paper each in EHMS within the period we reviewed. Although these two institutions are rated high in terms of quality medical research; however, this result is considerably low number compared to the result from Web of Science, this may possibly be connected to the fact that researchers tend to be interested more in Web of Science than Scopus, due to the international and multidisciplinary nature of the database to obtain literature in technology, science, medicine and other fields (Chadegani et al., 2013).

Table 4e Most productive and influential countries in EHMS (Web of Science)

\begin{tabular}{lccccc}
\hline Order & Country & Total paper & Total citation & h-index & $\begin{array}{c}\text { Average citations } \\
\text { per item }\end{array}$ \\
\hline 1 & USA & 43 & 1060 & 21 & 24.65 \\
3 & UK & 11 & 492 & 9 & 44.7 \\
5 & Canada & 9 & 162 & 7 & 18 \\
2 & China & 6 & 112 & 6 & 18.67 \\
13 & Spain & 3 & 25 & 3 & 8.33 \\
4 & Israel & 2 & 60 & 2 & 30 \\
8 & Germany & 2 & 41 & 2 & 20.5 \\
9 & France & 2 & 26 & 2 & 13 \\
10 & Australia & 2 & 30 & 2 & 15 \\
6 & Hong Kong & 1 & 45 & 1 & 45 \\
7 & South Korea & 1 & 25 & 1 & 25 \\
11 & Austria & 1 & 19 & 1 & 19 \\
12 & Ireland & 1 & 12 & 1 & 12 \\
14 & Taiwan & 1 & 10 & 1 & 10 \\
15 & Finland & 1 & 10 & 1 & 10 \\
16 & Brazil & 1 & 7 & 1 & 7 \\
17 & Italy & 1 & 6 & 1 & 6 \\
18 & Switzerland & 1 & 5 & 1 & 5 \\
19 & Algeria & 1 & 5 & 1 & 5 \\
20 & Greece & 1 & 5 & 1 & 5 \\
\hline
\end{tabular}

Table 4f Most productive and influential countries in EHMS (Scopus)

\begin{tabular}{lccccc}
\hline Order & Country & Total paper & Total citation & h-index & $\begin{array}{c}\text { Average citations } \\
\text { per item }\end{array}$ \\
\hline 1 & USA & 2 & 7 & 2 & 3.5 \\
2 & Finland & 1 & 5 & 1 & 5 \\
3 & Israel & 1 & 4 & 1 & 4 \\
4 & New Zealand & 1 & 2 & 1 & 2 \\
\hline
\end{tabular}

In terms of the most productive and influential countries in EHMS, USA is clearly the leading country in both Web of Science and Scopus based on our result. This analysis is 
detailed in Table 4c, where on Web of Science, USA takes the lead, followed by UK and Canada; USA also takes the lead again in Scopus. From our observation during the analysis, we noted that more about $47 \%$ of the institutions in our analysis are from the USA, $11 \%$ from the UK and $8 \%$ from Canada, making those three countries the most influential in EHMS. This is unsurprising, as these three identified countries are prominent in providing adequate funding and resources for medical research and implementation of new medical systems, periodic upskilling of medical practitioners in area of technology to ease usability of new systems, and organising regular sessions to raise awareness of new medical systems, which is having positive effects on the altitude, perception and adoption of new medical systems in the regions.

\subsection{Leading continents and sources in EHMS}

In Table 5, we have performed analysis of publications based on continent. The trend seems to be the same, as our result in both Web of Science and Scopus clearly shows that North America is the most productive region, with Europe and Asia taking the second and the third place respectively. However, taking into consideration paper per million inhabitants, the results are not so significant. Oceania and Africa are the least in EHMS in terms of productively, our result reveals that Africa has the lowest publication in EHMS on Web of Science and Scopus compared to other regions. Finally, Table 6 presents the most productive sources in EHMS, we have arranged this based on the total number of papers published in the sources from 2008 to 2018.

Table 5a Publication based on continent (Web of Science)

\begin{tabular}{lcccccccccc}
\hline Order & Continent & $T P$ & $T C$ & h-index & $C P$ & $\geq 250$ & $\geq 100$ & $\geq 50$ & $P M I$ & $C M I$ \\
\hline 1 & America & 53 & 1229 & 22 & 23.19 & 0 & 1 & 5 & 0.067 & 1.548 \\
2 & Europe & 24 & 641 & 12 & 26.71 & 0 & 2 & 3 & 0.032 & 0.858 \\
3 & Asia & 11 & 252 & 10 & 22.91 & 0 & 0 & 1 & 0.002 & 0.055 \\
4 & Oceania & 2 & 30 & 2 & 15.00 & 0 & 0 & 0 & 0.071 & 0.759 \\
5 & Africa & 1 & 5 & 1 & 5.00 & 0 & 0 & 0 & 0.001 & 0.004 \\
\hline
\end{tabular}

Note: Abbreviations: total paper - TP; total citation - TC; cites per paper - CP; paper per million inhabitants - PMI; cites per million inhabitants - CMI.

Table 5b Publication based on continent (Scopus)

\begin{tabular}{lcccccccccc}
\hline Order & Continent & $T P$ & $T C$ & h-index & $C P$ & $\geq 250$ & $\geq 100$ & $\geq 50$ & $P M I$ & $C M I$ \\
\hline 1 & America & 2 & 7 & 2 & 3.50 & 0 & 0 & 0 & 0.003 & 0.009 \\
2 & Europe & 1 & 5 & 1 & 5.00 & 0 & 0 & 0 & 0.001 & 0.007 \\
3 & Asia & 1 & 4 & 1 & 4.00 & 0 & 0 & 0 & 0.0002 & 0.0009 \\
4 & Oceania & 1 & 2 & 1 & 2.00 & 0 & 0 & 0 & 0.024 & 0.047 \\
5 & Africa & 0 & 0 & 0 & 0.00 & 0 & 0 & 0 & 0 & 0 \\
\hline
\end{tabular}

Note: Abbreviations: total paper - TP; total citation - TC; cites per paper - CP; paper per million inhabitants - PMI; cites per million inhabitants - CMI. 
Table 6a Most productive sources in EHMS (Web of Science)

\begin{tabular}{|c|c|c|c|}
\hline Order & Source title & $\begin{array}{l}\text { Total } \\
\text { paper }\end{array}$ & $\begin{array}{l}\text { Impact } \\
\text { factor }\end{array}$ \\
\hline 1 & $\begin{array}{l}\text { JOURNAL OF THE AMERICAN MEDICAL INFORMATICS } \\
\text { ASSOCIATION }\end{array}$ & 13 & 4.292 \\
\hline 2 & INTERNATIONAL JOURNAL OF MEDICAL INFORMATICS & 7 & 2.731 \\
\hline 3 & BMC MEDICAL INFORMATICS AND DECISION MAKING & 7 & 2.067 \\
\hline 4 & JOURNAL OF BIOMEDICAL INFORMATICS & 5 & 2.95 \\
\hline 5 & JOURNAL OF MEDICAL SYSTEMS & 5 & 2.415 \\
\hline 6 & QUALITY \& SAFETY IN HEALTH CARE & 2 & 2.16 \\
\hline 7 & BMJ OPEN & 2 & 2.376 \\
\hline 8 & BMC HEALTH SERVICES RESEARCH & 2 & 1.932 \\
\hline 9 & PLOS ONE & 2 & 2.776 \\
\hline 10 & ACADEMIC PEDIATRICS & 2 & 2.537 \\
\hline 11 & COMPUTER METHODS AND PROGRAMS IN BIOMEDICINE & 2 & 3.424 \\
\hline 12 & BMJ-BRITISH MEDICAL JOURNAL & 1 & 27.604 \\
\hline 13 & MEDICAL CARE RESEARCH AND REVIEW & 1 & 2.577 \\
\hline 14 & CANADIAN FAMILY PHYSICIAN & 1 & 2.186 \\
\hline 15 & JOURNAL OF GENERAL INTERNAL MEDICINE & 1 & 4.606 \\
\hline 16 & JOURNAL OF HOSPITAL INFECTION & 1 & 3.704 \\
\hline 17 & MOUNT SINAI JOURNAL OF MEDICINE & 1 & 1.623 \\
\hline 18 & MEDICAL CARE & 1 & 3.795 \\
\hline 19 & $\begin{array}{l}\text { IEEE TRANSACTIONS ON INFORMATION TECHNOLOGY IN } \\
\text { BIOMEDICINE }\end{array}$ & 1 & 2.493 \\
\hline 20 & INFORMATION SYSTEMS RESEARCH & 1 & 2.457 \\
\hline 21 & ACADEMIC MEDICINE & 1 & 5.083 \\
\hline 22 & CIN-COMPUTERS INFORMATICS NURSING & 1 & 1.029 \\
\hline 23 & ARTIFICIAL INTELLIGENCE IN MEDICINE & 1 & 3.574 \\
\hline 24 & DIABETES \& METABOLISM & 1 & 3.263 \\
\hline 25 & TECHNOLOGY AND HEALTH CARE & 1 & 0.787 \\
\hline 26 & EUROPEAN SURGERY-ACTA CHIRURGICA AUSTRIACA & 1 & 0.483 \\
\hline 27 & METHODS OF INFORMATION IN MEDICINE & 1 & 1.024 \\
\hline 28 & AMERICAN JOURNAL OF HYPERTENSION & 1 & 2.53 \\
\hline 29 & $\begin{array}{l}\text { INTERNATIONAL JOURNAL OF COMPUTER ASSISTED } \\
\text { RADIOLOGY AND SURGERY }\end{array}$ & 1 & 2.155 \\
\hline 30 & IEEE ACCESS & 1 & 4.098 \\
\hline 31 & SUSTAINABLE CITIES AND SOCIETY & 1 & 4.624 \\
\hline 32 & $\begin{array}{c}\text { CANADIAN JOURNAL OF ANESTHESIA-JOURNAL CANADIEN } \\
\text { D ANESTHESIE }\end{array}$ & 1 & 3.374 \\
\hline 33 & COMPUTERS IN BIOLOGY AND MEDICINE & 1 & 2.286 \\
\hline 34 & MEDICINA CLINICA & 1 & 1.277 \\
\hline 35 & ANAESTHESIA AND INTENSIVE CARE & 1 & 1.358 \\
\hline
\end{tabular}


Table 6a Most productive sources in EHMS (Web of Science) (continued)

\begin{tabular}{|c|c|c|c|}
\hline Order & Source title & $\begin{array}{l}\text { Total } \\
\text { paper }\end{array}$ & $\begin{array}{l}\text { Impact } \\
\text { factor }\end{array}$ \\
\hline 36 & ACM COMPUTING SURVEYS & 1 & 6.131 \\
\hline 37 & HEALTH POLICY AND TECHNOLOGY & 1 & 1.225 \\
\hline 38 & $\begin{array}{l}\text { INTERNATIONAL JOURNAL OF HEALTH POLICY AND } \\
\text { MANAGEMENT }\end{array}$ & 1 & 4.485 \\
\hline 39 & CLINICAL ORTHOPAEDICS AND RELATED RESEARCH & 1 & 4.154 \\
\hline 40 & KNOWLEDGE-BASED SYSTEMS & 1 & 5.101 \\
\hline 41 & EPILEPSY \& BEHAVIOR & 1 & 2.378 \\
\hline 42 & $\begin{array}{l}\text { ACM TRANSACTIONS ON COMPUTER-HUMAN } \\
\text { INTERACTION }\end{array}$ & 1 & 1.734 \\
\hline 43 & PERSONALIZED MEDICINE & 1 & 1.414 \\
\hline 44 & JOURNAL OF MEDICAL INTERNET RESEARCH & 1 & 4.945 \\
\hline 45 & NURSING PHILOSOPHY & 1 & 1.071 \\
\hline 46 & $\begin{array}{l}\text { IEEE JOURNAL OF BIOMEDICAL AND HEALTH } \\
\text { INFORMATICS }\end{array}$ & 1 & 4.217 \\
\hline 47 & APPLIED CLINICAL INFORMATICS & 1 & 1.306 \\
\hline 48 & AMERICAN JOURNAL OF PREVENTIVE MEDICINE & 1 & 4.435 \\
\hline 49 & JOURNAL OF HEALTHCARE ENGINEERING & 1 & 1.295 \\
\hline 50 & HEALTH POLICY & 1 & 2.075 \\
\hline 51 & JOURNAL OF PEDIATRIC INTENSIVE CARE & 1 & - \\
\hline 52 & SOCIAL SCIENCE \& MEDICINE & 1 & 3.087 \\
\hline 53 & PERSONAL AND UBIQUITOUS COMPUTING & 1 & 1.735 \\
\hline
\end{tabular}

Table 6b Most productive sources in EHMS (Scopus)

\begin{tabular}{lccc}
\hline Order & Source title & $\begin{array}{c}\text { Total } \\
\text { paper }\end{array}$ & $\begin{array}{c}\text { Impact } \\
\text { factor }\end{array}$ \\
\hline 1 & Proceedings -2011 International Conference on Cyber-Enabled & 1 & - \\
& Distributed Computing and Knowledge Discovery, CyberC 2011 & & \\
2 & Telemedicine and e-Health & 1 & 1.996 \\
3 & Sociology of Health and Illness & 1 & 2.211 \\
4 & Medical Data Privacy Handbook & 1 & - \\
5 & Journal of Information and Knowledge Management & 1 & - \\
\hline
\end{tabular}

Our result reveals that Journal of the American Medical Informatics Association is the most productive source on Web of Science, followed by International Journal of Medical Informatics and BMC Medical Informatics and Decision Making taking the second and third place. We have also included in our analysis the impact factors of these sources at the time of our study and we have identified few observations below:

- Quality and Safety in Health Care, which was ranked no. 6 in terms of productivity based on our analysis, it had impact factor of 2.160 on Web of Science and this was shown as being updated last in 2012. However, the information is different on the journal homepage, the impact factor was noted as 7.043 . 
- Mount Sinai Journal of Medicine was ranked no. 17 in terms of productivity (based on our analysis), it had impact factor of 1.623 on Web of Science and this was shown as being updated last in 2014.

- IEEE Transaction on Information Technology in Biomedicine was ranked no. 19 in terms of productivity (based on our analysis), it had impact factor of 2.493 on Web of Science and this was shown as being updated last in 2014.

Proceedings of 2011 International Conference on Cyber-Enabled Distributed Computing and Knowledge Discovery, Telemedicine and e-Health are the leading in terms of most productive sources in EHMS on Scopus.

\section{Graphical analysis of EHMs with VOSviewer}

The study runs a bibliometric analysis with VOSviewer version 1.6.13 (Van Eck, \& Waltman 2010) to create bibliometric maps with items, links, and clusters for the purpose of identifying the milestones in research area of EHMS and to examine the areas that makes up EHMS and how they connect to each other. This study extracted data from two bibliographic databases of Web of Science and Scopus that contains the metadata of authors, year of publications, cited references, affiliations, source, volume, issue, pages, digital object identifier (DOI), abstract, keywords and document type for comprehensive analysis and comparison. The study based on the recommendation of (Van Eck and Waltman, 2010) also examined the co-citation of journals, a bibliographic coupling of authors, bibliographic coupling of institutions, citation analysis of institutions, co-authorship of institutions, a bibliographic coupling of countries and co-occurrence of author keywords.

It is important to understand how co-citation works and its application in this study. Co-citation harmonises the two items that are published in separate journals that receive a citation from another journal item. In Figures 2(a) and 2(b), this study has the graphical representation of journals co-citation between 2008 to 2018 from both Web of Science and Scopus. For Web of Science, we set the threshold to 85 and out 1,548 sources, only three sources meet the set threshold. To expand the scope of the sources, we set reduced the threshold to 2 and out of 1548 , only 341 sources meet the threshold and the analysis with two threshold reveals 11 clusters of sources and the first clusters records 50 items. In all, the Journal of the American Medical Informatics Association had the total link strength of 11,286. In comparison with Scopus map network, there are distant differences in the weight of sources of Web of Science regarding EHMS based on clusters but on the part of Scopus, there was no distinct demarcation of the sources weight but the Scopus map network link was dense with some sources at the borders [Figures 2(a) and 2(b)]. 
Figure 2 (a) Co-citation of journals cited in EHMS (Web of Science) (b) Co-citation of journals cited in EHMS (Scopus) (see online version for colours)

epilepsia

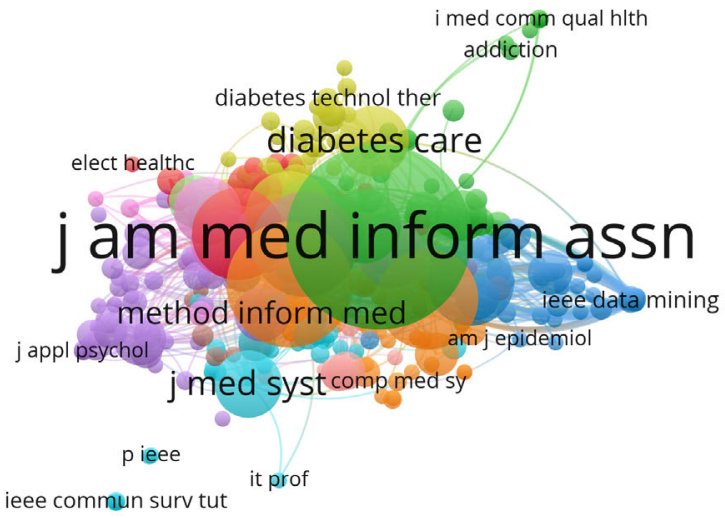

\& VOSviewer

(a)

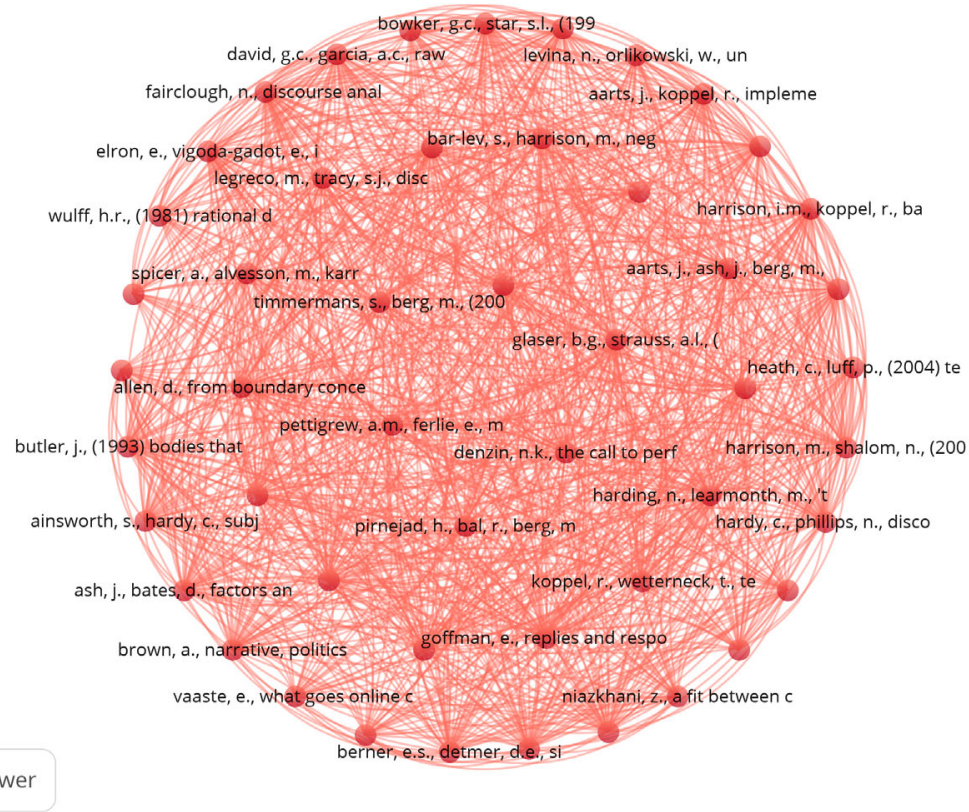

(b) 
Figure 3 (a) Bibliographic coupling of authors that published in EHMS (Web of Science)

(b) Bibliographic coupling of authors that published in EHMS (Scopus)

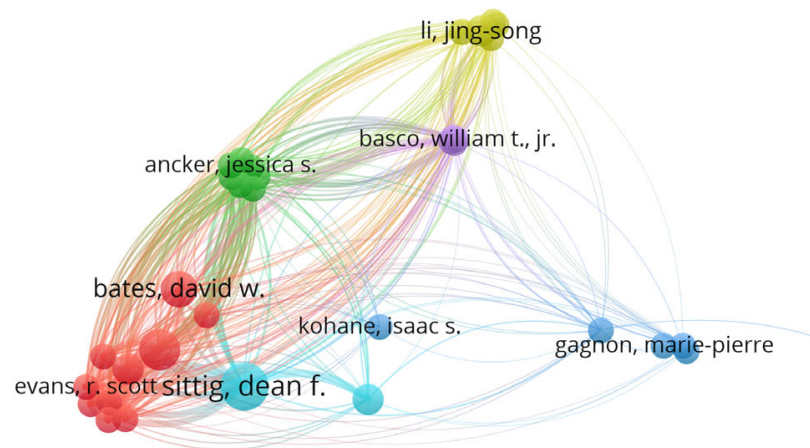

\section{\& Vosviewer}

(a)

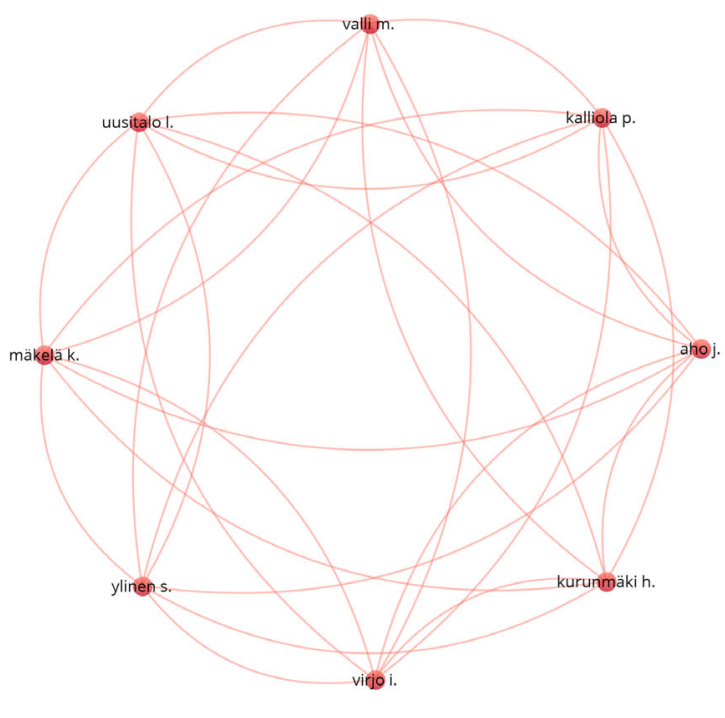

(b)

We compare bibliographic coupling of authors in Figures 3(a) and 3(b). We used the thresholds of 4 and out of 477 authors, 3 meets the set thresholds. We also used the threshold of 2 , and only 34 authors meet the criteria. The higher the thresholds, the lower the number of authors that reaches the limit. The analysis shows six clusters and the first cluster had ten items. Among the 34 authors, Sittig, Dean and Singh, Hardeep had total link strength more than the other authors. The network map of Web of Science in the field of EHMS is more densely than Scopus network map [Figures 3(a) and 3(b)]. 
Regarding bibliographic coupling of institutions, four thresholds shows that out of 233 institutions, five meet the thresholds while two thresholds shows that 34 meets the thresholds with seven clusters and the first cluster records eleven items. Harvard University had the highest total link strength of 1,650, which indicate the relatedness between two items. Some institutions in Web of Science clustered closely while the institutions in Scopus have distance relatedness [Figures 4(a) and 4(b)].

Figure 4 (a) Bibliographic coupling of institutions that published in EHMS (Web of Science) (b) Bibliographic coupling of institutions that published in EHMS (Scopus) (see online version for colours)

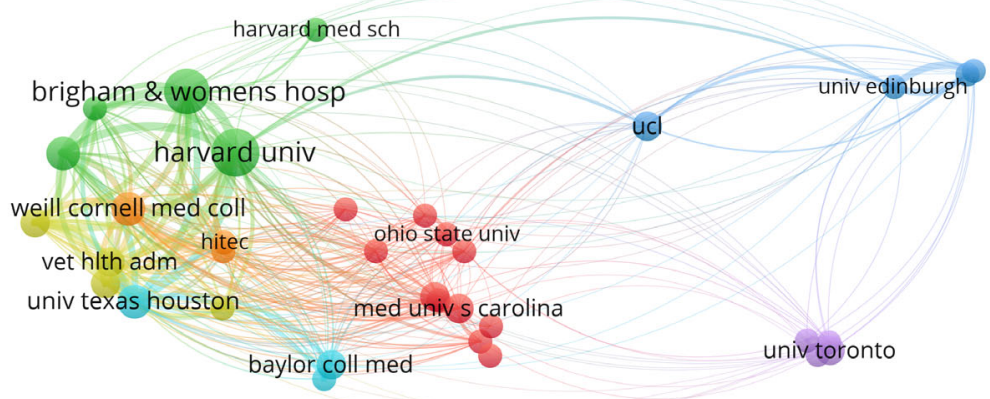

\section{\& $\mathrm{c}$ VOSviewer}

(a)

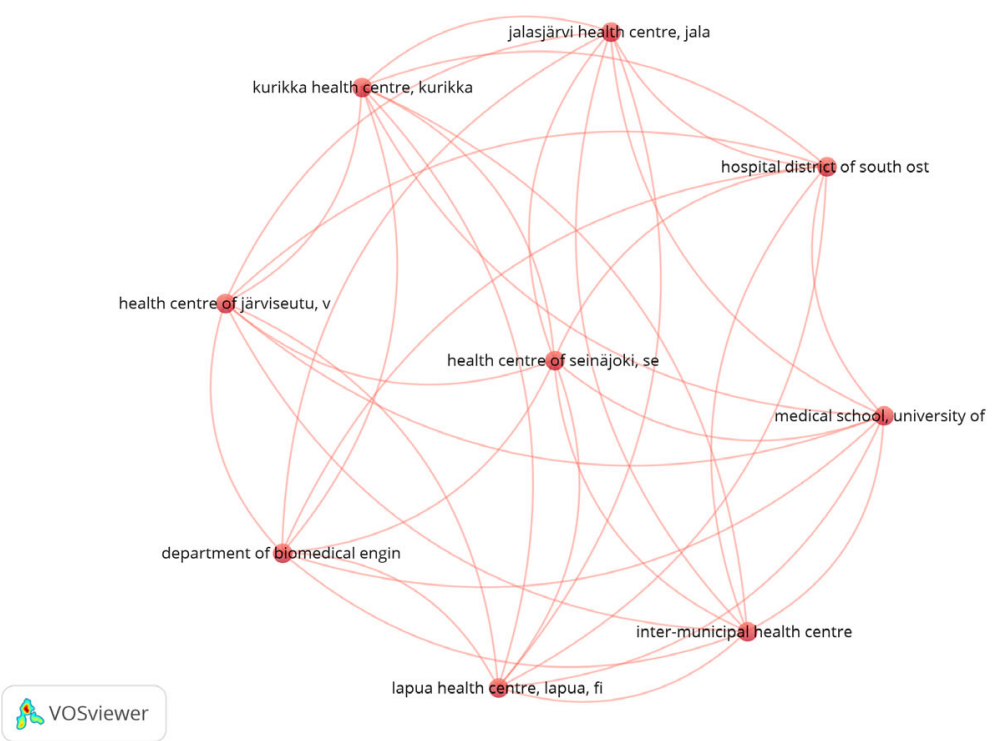

(b) 
Figure 5 (a) Citation analysis of countries that published in EHMS (Web of Science) (b) Citation analysis of countries that published in EHMS (Scopus) (see online version for colours)

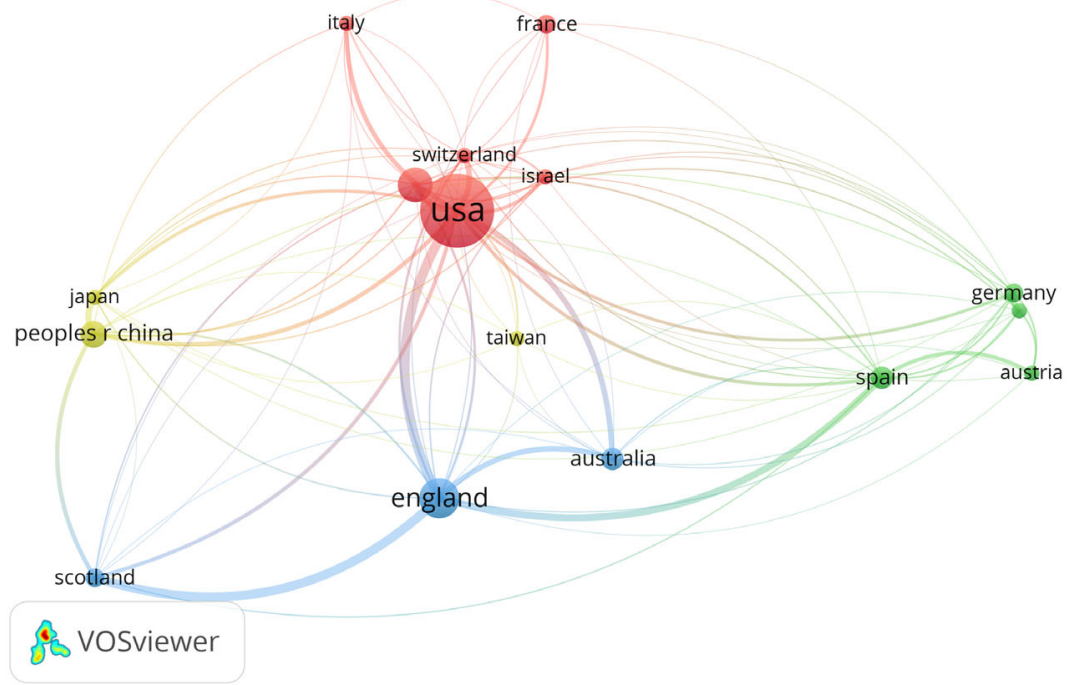

(a)

new zealand

united states

finland

VOSviewer

israel

(b)

As shown in Figures 5(a) and 5(b), the Web of Science output for EHMS with thresholds of 10 shows that out of the 28 countries generated, three countries reached the thresholds while with 2 thresholds, 16 countries conforms to the set thresholds. The Vosviewer generate four clusters and the first cluster reveals six items. The six countries in cluster one falls to North America, Europe and Israel (Middle East). The USA had the highest 
citations with 9 total link strength. Unlike Web of Science link strength, the Scopus had four clusters without any link strength.

Figure 6 (a) Co-authorship of institutions that published in EHMS (Web of Science)

(b) Co-authorship of institutions that published in EHMS (Scopus) (see online version for colours)

ohio state univ

univ alberta

zhejiang univ

univ cincinnati

univ edinburgh

harvard univ

baylor coll med

univ london london sch econ \&

univ toronto

kaiser permanente

\& Vosviewer

med univs carolina

(a)

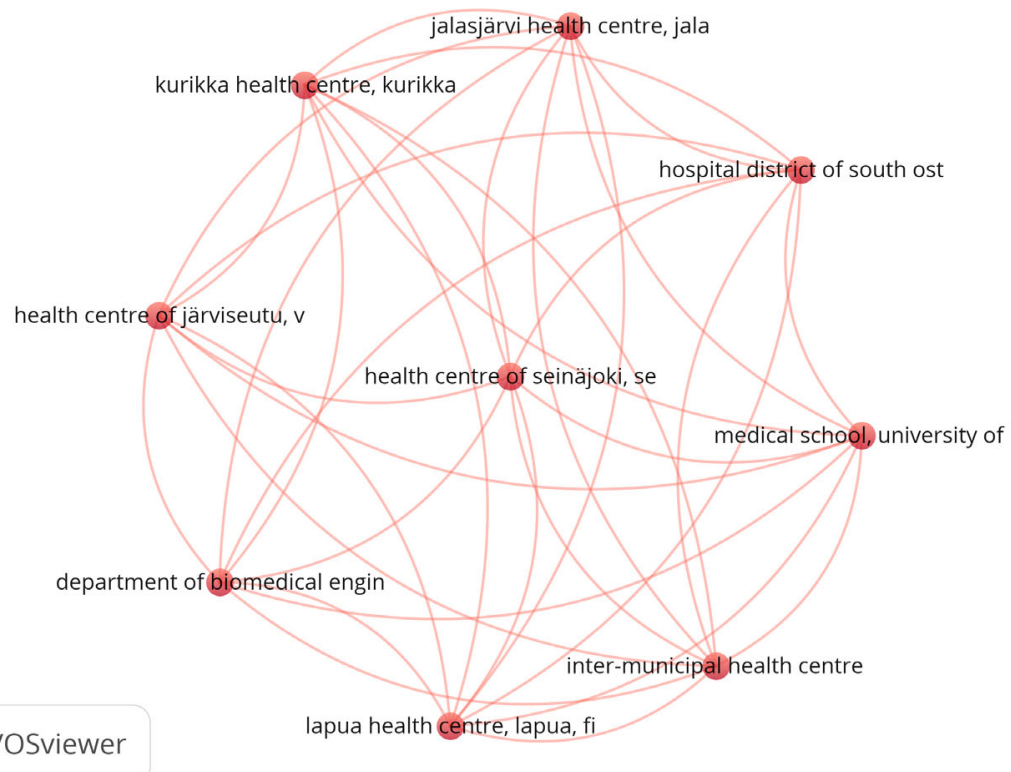

(b) 
Initially, we set the thresholds of 4 and out of 233 institutions, five meet the thresholds in Web of Science. We also tried the thresholds of 2 and out of the 233 institutions, 34 meet the thresholds. The 34 institutions formed 13 clusters and the highest densely cluster had five items and they consists Harvard University, UCI, University of Edinburgh, University of Leeds and University of London Imperial College. The co-authorship of institutions is a combination of clustered institutions with link strengths and institutions without link strengths. The Scopus co-authorship of institutions map network were connected with uniform weights.

Table 7a Most frequent author's keyword in EHMS (Web of Science)

\begin{tabular}{ccl}
\hline Cluster & No. of items & \multicolumn{1}{c}{ Author keywords } \\
\hline 1 & 7 & $\begin{array}{l}\text { decision-support, impact, implementation, information-technology, } \\
\text { physician order entry, quality, systems } \\
\text { electronic health records, information, medical-records, prevalence, } \\
\text { primary-care, risk }\end{array}$ \\
3 & 6 & $\begin{array}{l}\text { adoption, electronic medical records, health-care, hospitals, } \\
\text { physicians } \\
\text { care, electronic health record, management, technology }\end{array}$ \\
\hline
\end{tabular}

Table 7b Most frequent author's keyword in EHMS (Scopus)

\begin{tabular}{lcl}
\hline Cluster & No. of items & \multicolumn{1}{c}{ Author keywords } \\
\hline 1 & 2 & electronic health records, electronic medical records \\
2 & 1 & electronic health record \\
\hline
\end{tabular}

Lastly, we examine the co-occurrence of author keywords and all keywords of health records in Web of Science and author keywords in Scopus in Figures 7(a), 7(b), and 7(c). Using 6 as thresholds for authors keywords analysis, out of 301 keywords generated, only 3 meets the thresholds and while using the thresholds of 2, out of the 301 keywords, only 26 reach the set verge, forming 8 clusters and the first cluster had 6 items. In all keywords, two thresholds reveal 568 keywords shows 109 keywords that the meet the set thresholds with 7 clusters and the first clusters with 32 items. In author's keywords, electronic health records had the highest total link strength, followed by electronic health record with 11 total link strength, health information technology with 8 total link strength, electronic medical records and semantic interoperability with 7 total link strength. For all keywords, management had 121 total link strength, followed by information-technology with 111 total link strength, electronic health records with 102, physicians with 61, implementation with 58. Physician order entry with 38 , electronic health record and decision-support with 37 total link strength. For Scopus, only three keywords emerged and they electronic medical record, electronic health records, organisation and management. Management and electronic health records are common in the Web of Science and Scopus. 
Figure 7 (a) Co-occurrence of author keywords of documents published in EHMS (Web of Science) (b) Co-occurrence of author keywords of documents published in EHMS (Web of Science) (c) Co-occurrence of author keywords of documents published in EHMS (Scopus) (see online version for colours)

machine learning

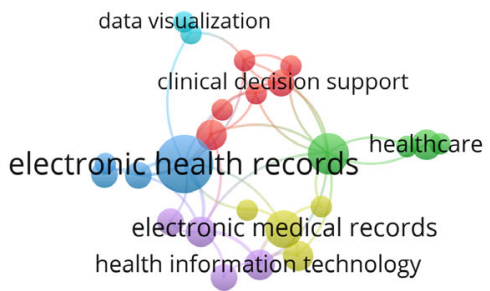

\& Vosviewer

electronic medical record (emr

(a)

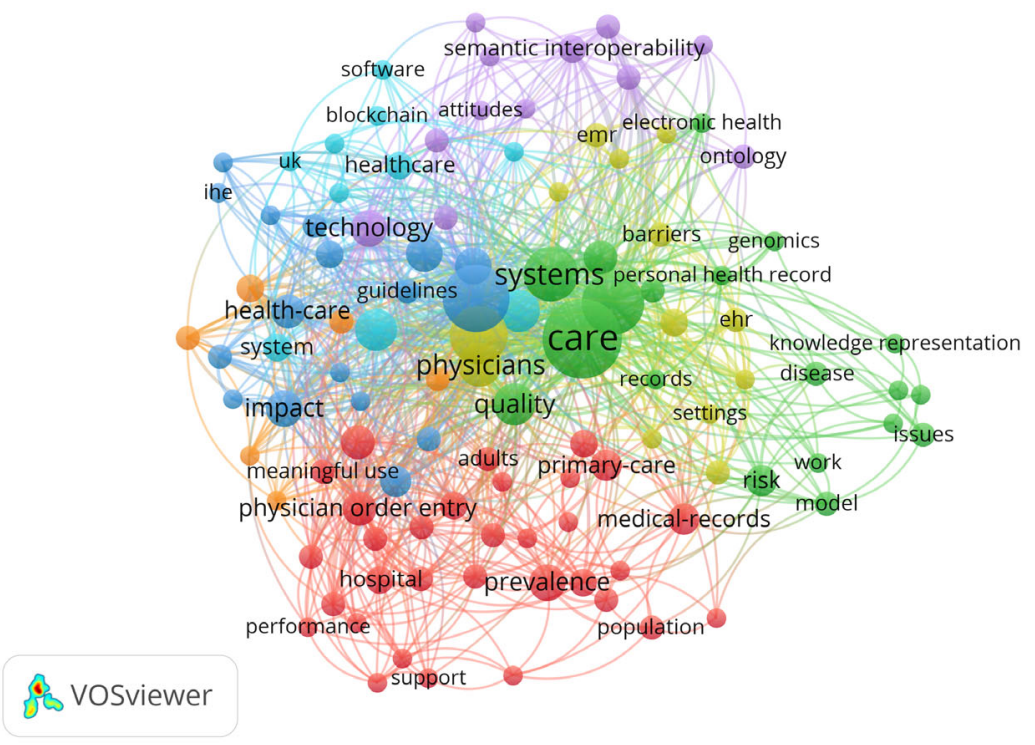

(b) 
Figure 7 (a) Co-occurrence of author keywords of documents published in EHMS (Web of Science) (b) Co-occurrence of author keywords of documents published in EHMS (Web of Science) (c) Co-occurrence of author keywords of documents published in EHMS (Scopus) (continued) (see online version for colours)

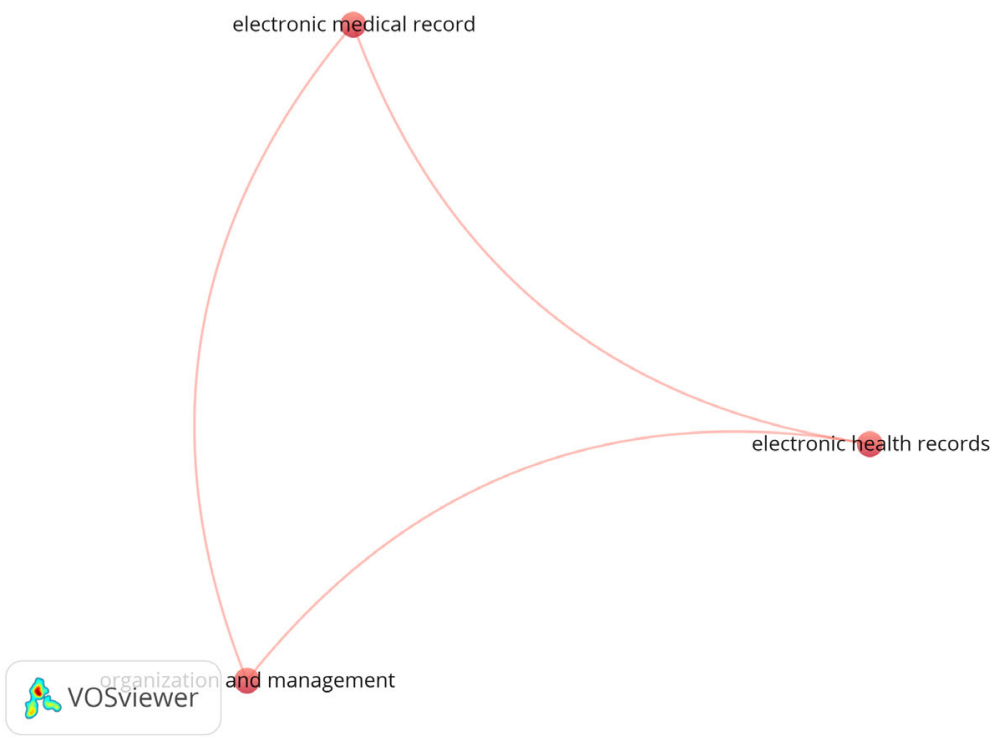

(c)

\section{Summary and conclusions}

This study searched for publications related to EHMS in two databases (Web of Science and Scopus) between 2008-2018, We selected 96 EHMS related papers in total out of 508 publications found (91 were from Web of Science and five from Scopus). Particularly, it is worth noting that this study found only a relatively small number of literatures focused on EHMS adoption, influence and implementation. This lack of research literature and relevant information may have contributed largely to the slow adoption and implementation of EHMS globally, which is more notable in Africa and Oceania from our analysis and result. Our results provide insight into the research and publication trends on the implementation, influence, and adoption of EHMS over the period reviewed, and we have been able to demonstrate with our results how research in this area have evolved over time.

In analysing our research result from the bibliometric analysis of the 96 EHMS related papers, we noted that Wright, Adam has three papers in this review, making him the most productive author in EHMS on WoS based on our ranking on the number of publications for each author. Sittig, Dean; Ancker, Jessica; Li, Jing-Song and Teufel, Ronald; all had two papers each. Chang and Makela have been ranked as the most 
productive author in Scopus in EHMS within the period we have reviewed with one paper each. The three most cited papers in Web of Science were written by Sittig, Dean; Greenhalgh, Trisha and Lluch, Maria with 171, 143 and 117, respectively. In Scopus, Chang; Makela, Virjo et al. and Bar-Lev represent the top-three most cited papers in EHMS.

It is worth noting that the yearly citation trend from Web of Science (WoS) reveals that 2010 and 2012 had the highest number of citations in EHMS with a total of 8 and 14 papers respectively and all the publications in Scopus on EHMS were published between 2010-2015. Our result also reveals that the numbers of citations and papers in EHMS is declining through time with 2017 seeing the least citations within the period under review. On Scopus, 2015 had the highest number of citations with a total of two papers, followed by 2010 and 2011 who both had five citations each. Our results show that there has been no paper published in EHMS since 2008-2009 and between 2016-2018 in Scopus.

It is also worth noting that our result further reveals Harvard medical school as the most productive institution in WoS; surprisingly, Wright, Adam who is the most productive author in WoS is affiliated to this institution; Zhejiang University and Medical University of South Carolina took the second and third places. We presumed that productivity of these institutions could have been as a result of dedicating more resources to medical research in their respective regions. Although Lawrence Technological University and Tampere University of Technology were observed as the most productive institutions in EHMS on Scopus with one paper each. We noted that this result is considerably low number compared to the result from Web of Science, this may possibly be connected to the fact that researchers tend to be interested more in Web of Science than Scopus, due to the international and multidisciplinary nature of the database to obtain literature in technology, science, medicine and other fields (Chadegani et al., 2013).

The most influential country in EHMS from WoS and Scopus based on our review was USA; interestingly, the first three most productive authors were affiliated to institutions in the USA. This is followed by UK and Canada being the second and third most productive countries. These three identified countries are prominent in providing adequate funding and resources for medical research, periodically upskilling their medical practitioners in area of technology and organising regular sessions to raise necessary awareness of new medical tools, which we believe is having positive effects on the altitude, perception and adoption of new medical systems in the regions.

Based on our result, North America is the most productive continent on EHMS, which has more than $58 \%$ of the total publications reviewed on EHMS in our study. Europe and Asia taking the second and the third place respectively. Oceania and Africa are the least influential continents in EHMS, which raise a major concern on the need to urgently investigate what constitute barriers in these regions in terms of EHMS.

In order to further expand on our bibliometric results, our study also develops a graphical visualisation of the results using the VOS viewer software, which shows the publication structure of authors, institutions, and countries, by using bibliographic coupling, co-authorship and citation analysis. The results agree with our section 4 , where USA is clearly the most productive country. 
As a limitation of this study, we have limited our search to only publications between 2008-2018 in the two databases; hence our study does not take into consideration years beyond this period. In addition, using 'English' as a limit during the search meant that we may have excluded key literatures on EHMS adoption and implementation written in another languages or that may have been authored by researchers who resides in countries with high adoption and implementation of EHMS who are not 'English' speakers.

In conclusion, our study has revealed the need to develop a strong evidence base research to support the use, adoption, influence and effective implementation of EHMS in health care institutions.

\section{References}

Adebayo, K.J. and Ofoegbu, E.O. (2014) 'Issues on e-health adoption in Nigeria', International Journal of Modern Education and Computer Science, Vol. 6, No. 9, p.36.

Ajami, S. and Bagheri-Tadi, T. (2013) 'Barriers for adopting electronic health records (EHRs) by physicians', Acta Informatica Medica, Vol. 21, No. 2, p.129.

Borgman, C.L. (1989) 'Bibliometrics and scholarly communication: editor's introduction', Communication Research, Vol. 16, No. 5, pp.583-599.

Broadus, R. (1987) 'Toward a definition of 'bibliometrics', Scientometrics, Vol. 12, Nos. 5-6, pp.373-379.

Chadegani, A.A., Salehi, H., Yunus, M., Farhadi, H., Fooladi, M., Farhadi, M. and Ebrahim, N.A. (2013) 'A comparison between two main academic literature collections: Web of Science and Scopus databases', Asian Social Science, Vol. 9, No. 5, pp.18-26.

Clarke, M. and Horton, R. (2001) 'Bringing it all together: Lancet-Cochrane collaborate on systematic reviews', The Lancet, Vol. 357, No. 9270, p.1728.

Evans, R.S. (2016) 'Electronic health records: then, now, and in the future', Yearbook of Medical Informatics, Vol. 25, No. S01, pp.S48-S61.

Nicholas, D. and Ritchie, M. (1978) Literature and Bibliometrics, C. Bingley, London.

Pérez-Jover, V., Sala-González, M., Guilabert, M. and Mira, J.J. (2019) 'Mobile apps for increasing treatment adherence: systematic review', Journal of Medical Internet Research, Vol. 21, No. 6, p.e12505.

Podsakoff, P.., MacKenzie, S.B., Podsakoff, N.P. and Bachrach, D.G. (2008) 'Scholarly influence in the field of management: A bibliometric analysis of the determinants of university and author impact in the management literature in the past quarter century', Journal of Management, Vol. 34, No. 4, pp.641-720.

Pritchard, A. (1969) 'Statistical bibliography or bibliometrics', Journal of Documentation, Vol. 25, No. 4, pp.348-349.

Salehahmadi, Z. and Hajialiasghari, F. (2013) 'Telemedicine in Iran: chances and challenges', World Journal of Plastic Surgery, Vol. 2, No. 1, p.18.

Small, H. (1973) 'Co-citation in the scientific literature: A new measure of the relationship between two documents', Journal of the American Society for Information Science, Vol. 24, No. 4, pp.265-269.

Stevens, R.E. (1953) Characteristics of Subject Literatures, Publications Committee of the Association of College and Reference Libraries, Chicago. 
Tranfield, D., Denyer, D. and Smart, P. (2003) 'Towards a methodology for developing evidence-informed management knowledge by means of systematic review', British Journal of Management, Vol. 14, No. 3, pp.207-222.

Van Eck, N. and Waltman, L. (2009) 'Software survey: VOSviewer, a computer program for bibliometric mapping', Scientometrics, Vol. 84, No. 2, pp.523-538.

Welsh, T.S. (2017) 'Qualitative and quantitative methods in libraries journal special issue: bibliometrics and scientometrics', Qualitative and Quantitative Methods in Libraries, pp.1-3, July, ISSN 2241-1925 [online] http://www.qqml-journal.net/index.php/qqml/article/view/357. 